\title{
A computational model of aesthetic value
}

Aenne A. Brielmann

\author{
Peter Dayan
}

\begin{abstract}
August 2021
Abstract

People invest precious time and resources on experiences such as watching movies or listening to music. Yet, we still have a poor understanding of how such sensed experiences gain aesthetic value. We propose a model of aesthetic value that integrates existing theories with literature on conventional primary and secondary rewards such as food and money. We assume that the states of observers' sensory and cognitive systems adapt to process stimuli effectively in both the present and the future. These system states collectively comprise a probabilistic generative model of stimuli in the environment. Two interlinked components generate value: immediate sensory reward and the change in expected future reward. Immediate sensory reward is taken as the fluency with which a stimulus is processed, quantified by the likelihood of that stimulus given an observer's state. The change in expected future reward is taken as the change in fluency with which likely future stimuli will be processed. It is quantified by the change in the divergence between the observer's system state and the distribution of stimuli that the observer expects to see over the long term. Simulations show that a simple version of the model can account for empirical data on the effects of exposure, complexity, and symmetry on aesthetic value judgments. Taken together, our model melds processing fluency theories (immediate reward) and learning theories (change in expected future reward). Its application offers insight as to how the interplay of immediate processing fluency and learning gives rise to aesthetic value judgments.
\end{abstract}

keywords: aesthetic value, reward, processing fluency, learning

Draft version 2.0, 05/08/21.

This paper has not (yet) been published and is not therefore the authoritative document of record. Please do not copy or cite without author's permission. 


\section{Introduction}

Cynics, or perhaps economists, are said to know the price of everything, but the value of nothing. However, when it comes to money, at least, economists have highly developed systems for understanding aspects of its value. Thus, although money's absolute subjective value poses quantification problems, these values generally obey at least some reasonable laws - e.g., that $\$ 2$ is more valuable than $\$ 1$ for individuals to a degree that can be related to their measurable characteristics (such as income) or concrete experienced inputs (such as gains and losses in previous trials). Indeed, lawlike behaviour is sufficiently common that questions about law-breaking can be of considerable academic interest (Kahneman, 2011).

In contrast, economists and aestheticians alike struggle to provide an account of the value of sensed objects. These so-called aesthetic values characterize the pleasure derived from a sensed experience. They are a key component of our everyday lives and decision-making. Whether it be the clothes we wear, the places we live, or the people we fall in love with, the pleasantness of their sensed characteristics help determine which ones we chose. Despite this importance, we still only have a poor understanding of how the sensed properties of an object become a source of pleasure, i.e., how they accquire aesthetic value. This stands in contrast to other kinds of rewards, like money or nutrition, for which clearly defined, measurable properties (currency value, calories) provide a starting point for assessing their reward value. For aesthetic values, however, we even lack a reliable means of quantification other than self-report. Thus, even though reward derived from sensed experience and other kinds of reward share at least some underlying mechanisms (Brown et al., 2011; Kühn \& Gallinat, 2012; Mas-Herrero et al., 2021), the fact that the value of sensed experience is generated purely inside the observer has posed considerable challenges to its scientific investigation.

Despite these difficulties, the field of experimental aesthetics has accumulated data that document certain regularities in people's aesthetic judgments (see, e.g. Brielmann and Pelli, 2018; Palmer et al., 2013). Furthermore, many of these empirical findings have been, separately, linked to theoretical frameworks. We will briefly review the best-documented empirical findings and the two main families of theories that account for some known regularities in people's aesthetic reports and choices, but fail to encompass other intuitions and data. Then, we will propose a new computational model that quantifies the aesthetic value of an object by drawing on the ideas of both families of theories.

It is important to set the terminological table at the outset. We consider aesthetic value to be the basis of a variety of aesthetic evaluations, such as (dis-)liking, (dis-)pleasure, or beauty, as well as of aesthetic preferences and choices. Our theory and model exclusively relate to this narrow definition of aesthetic value. They do not extend to a broader concept of aesthetic value that includes the entire spectrum of possible evaluative responses one may have in response to an aesthetic experience and are targeted by accounts such as The Vienna Integrated Model of topdown and bottom-up processes in Art Perception (Pelowski et al., 2017), or Leder and Nadal's model of aesthetic appreciation and aesthetic judgments (Leder \& Nadal, 2014).

Evaluation, judgment, preference, etc. are often used interchangeably throughout the literature. However, absolute aesthetic evaluations of a single object (the Mona Lisa is a 6 out of 7 on the beauty-scale) are different from relative statements of preference (the Mona Lisa is more beautiful than The Starry Night) or choices (I want to look at the Mona Lisa, not The Starry Night). Further, the term "liking" itself has a very specific connotation in the context of a distinction be- 
tween "wanting" and "liking" (e.g., Berridge et al., 2009). Liking in this context means having a positive hedonic response while wanting means being willing to work towards obtaining an experience. A clear conceptual distinction between liking and wanting has, however, so far eluded a clear operationalization (Pool et al., 2016). To avoid terminological confusion, we will here refer to evaluations such as liking, pleasure, and beauty as aesthetic value judgments. These judgements will be the main focus of this article, although we also briefly discuss the implications of our theory for aesthetic preferences, choices, and a potential distinction between "liking" and "wanting".

\subsection{Existing empirical findings}

Research on aesthetic judgments is rapidly growing (Anglada-Tort \& Skov, 2020). We therefore limit our review of empirical findings to areas with substantial data that relate to core predictions of existing theories. This means that most of the research we consider will rely on visual or auditory stimuli, and the manipulation of few independent variables. Even though the scope of our model in principle extends to all sensory modalities, including olfaction, taste, and touch, it is beyond the scope of the current article to discuss these less well-established findings as well. And although they are of great interest, and well within the ambition of our theoretical approach, we pay little attention to the known, large individual differences between people's aesthetic judgments (e.g., Vessel et al., 2018), or differences between groups based on, e.g., relevant expertise or cultural background.

\subsubsection{Averageness and prototypicality}

People rate more average, or prototypical, exemplars of many different kinds of objects more positively than atypical ones, including words (Martindale et al., 1988), colors (Martindale \& Moore, 1988), chords (Brattico et al., 2009), consumer goods (Scarpi et al., 2019), abstract dot patterns (Vogel et al., 2018: Winkielman et al., 2006), animals and cars (Halberstadt \& Rhodes, 2003), and faces (Dotsch et al., 2016; Muñoz-Reyes et al., 2015; Rhodes et al., 2001; Ryali et al., 2020) with the potential exception of negatively valenced image categories (Vogel et al., 2020). In the case of faces, the effect is often attributed to an underlying positive valuation of smooth-skinned, symmetrical faces, supposedly indicating better, less damaged, genetic material (Rhodes, 2006).

\subsubsection{Symmetry}

All else being equal, the great majority of people prefer symmetric over asymmetric visual stimuli. This finding has been well-established using simple black-and-white patterns (Höfel \& Jacobsen, 2007; Jacobsen \& Höfel, 2002; Jacobsen et al., 2006; Rampone et al., 2016; Westphal-Fitch et al., 2012) - even though individual differences (Corradi et al., 2020) and art expertise moderate this symmetry effect (Gartus et al., 2020: Leder et al., 2018; Weichselbaum et al., 2018). People also find more symmetric faces more attractive (Mealey et al., 1999; Muñoz-Reyes et al., 2015; Rhodes et al., 2001) (though not artificially perfectly symmetrical ones (Zaidel \& Deblieck, 2007)) a finding that is linked to a general preference for more average faces (see above). 


\subsubsection{Complexity}

Complexity has been prominently featured in research on aesthetic judgments since the early beginnings of the subject (Berlyne, 1970; Birkhoff, 1933; Fechner, 1876). To date, the findings on the relation between complexity and aesthetic judgments are mixed, and this may be due to individual differences in the direction of the relation between complexity and aesthetic value judgments (Corradi et al., 2020; Jacobsen \& Höfel, 2002; Lyssenko et al.,2016; Sherman et al., 2015; Street et al., 2016), types of complexity manipulations (Christensen et al., 2020; Nadal et al., 2010), and stimulus type (Marin et al., 2016; Mayer \& Landwehr, 2018). Partially, these inconsistencies might be related to differences in subjectively perceived versus objectively measured complexity (Sherman et al., 2015). A recent review of contemporary studies suggests that the diverging findings may also partially be explained by an interaction between order, as e.g., introduced by symmetry, and complexity (Van Geert \& Wagemans, 2020). This argument resembles the principle of "unity in variety"(Fechner, 1876): the notion that various elements can be integrated into a uniform whole when arranged in a systematic manner.

\subsubsection{Mere exposure}

Ever since Zajonc's (1968) seminal report on the effects of repeatedly presenting the same stimulus, so-called "mere exposure" effects have drawn interest from numerous researchers who have produced a wealth of data documenting the effect. A recent comprehensive meta-analysis of this line of work (Montoya et al., 2017) concludes that the relation between the number of exposures and liking or pleasingness of an object follows a shallow inverted U-shape with a peak around 35 to 40 exposures. Intuitively, this reversal of the positive mere exposure effect corresponds to "boredom" (Geana et al., 2016). Upon closer inspection, results from the individual studies included in the meta-analysis reveal that repeated exposure can affect judgments in different ways and in some cases also lead to an asymptotic increase, U-shaped, or even monotonically decreasing relationship between the exposure and evaluation.

\subsubsection{Uncertainty and surprise}

The findings above rely mostly on studies that use static visual stimuli. The inherently dynamic nature of music has invited the investigation of other factors, most notably surprise (a deviation of an event from immediate expectations), and linked to it, uncertainty (lack of a deterministic expectation about an event before it occurs). Studies that looked at the effects of uncertainty and/or surprise have so far yielded mixed results. Surprising musical events in piano music can both increase and decrease continuously reported pleasure depending on the particular musical piece (Shany et al., 2019). Surprise in the form of syncopation (deviations from the meter) exhibits an inverted U-shaped relationship with self-reported pleasantness of funk music (Witek et al., 2014). Recent research that used the IDyOM algorithm (Pearce, 2005) to quantify uncertainty and surprise based on the statistical properties of a large corpus of Western music has shown that surprising chords are pleasant only when uncertainty is low (Cheung et al., 2019).

\subsubsection{Framing effects, titles, and other additional information}

Sensed object properties, even when taking an observer's history of previous experiences into account, cannot completely account for aesthetic value judgments. The context in which they 
are encountered matters, too. Congruent or elaborating titles increase aesthetic value judgments for artworks (Anglada-Tort et al., 2019; Gerger \& Leder, 2015; Leder et al., 2006; Millis, 2001; Mullennix \& Robinet, 2018). Information about an object's history of creation also alters aesthetic judgments: People judge the aesthetic values of originals higher than those of (identical) forgeries (Newman \& Bloom, 2012) and of works labeled as gallery exhibits higher than those labeled as being computer-generated (Kirk et al., 2009). People also judge images otherwise deemed ugly less negatively when they are labeled as art instead of non-art (Wagner et al., 2014), similar to the effect of brand (McClure et al., 2004) and price labels (Plassmann et al., 2008) on people's evaluations of drinks.

\subsubsection{Connections between various effects}

Of course, many of the variables discussed above are related to one another. Prototypical objects are less surprising; repeated exposure can (temporarily) alter prototypes; symmetry can reduce perceived complexity by introducing a form of order, and expertise can offset effects of objectively defined complexity (Zhang et al., 2020). To date, the hierarchy of the various effects we discussed above is rather unclear, as is the relative strength of each of their contributions. We do know, however, that these various effects interact: the effects of symmetry and complexity can, for instance, change depending on exposure (Tinio \& Leder, 2009). It is therefore all the more important to formulate a theoretical framework that can provide a unified explanation for these isolated findings.

\subsection{Current theories}

There are many individual theories about aesthetic judgment and valuation that partially address the empirical findings listed above. We focus on aesthetic value (and so do not further discuss the important theories of art appreciation; e.g., Chatterjee and Vartanian, 2016; Leder and Nadal, 2014; Pelowski et al., 2017). The relevant theories can be organized in various ways (e.g., Palmer et al., 2013); we consider two main families, one centred on fluency (also including prototype theories) and the other on learning (also including arousal dynamics; Berlyne, 1970, and IDyOM). We ultimately view fluency and learning theories as closely related. We formalize fluency in terms of what it is that the learning process described by learning theories is trying to achieve and derive a new, integrated, theory in which aesthetic value arises as a temporally sophisticated prediction error associated with estimates of the long-run future fluency of processing.

\subsubsection{Processing fluency theories}

Processing fluency theories rest on three core assumptions: (i) The sensory-cognitive system of an observer is particularly suited to processing objects with certain sensed features. (ii) There is a neural signal that indicates how well the system is suited to the features of the current sensed input. (iii) This signal automatically triggers an affective response that is more positive, the better the sensory system suits the current sensed input.

For human observers, the most frequently discussed indicator of such a match between sensed input and the setting of the sensory-cognitive system is so-called "processing fluency". Processing fluency is considered to signal "progress toward successful recognition of the stimulus, error-free processing, or the availability of appropriate knowledge structures to interpret the stimulus ... [or] that an external stimulus is familiar ..." (Reber et al., 2004). In one sense, the processing fluency 
signal can be thought of as an a priori metacognitive signal that indicates how straightforward the observer expects processing to be based on stimulus properties and past experiences and that arises before the stimulus has been fully processed. This anticipatory fluency signal resembles the initial evaluation of processing success preceding 'Aha' experiences (Dubey et al., 2021; Muth \& Carbon, 2013: Van de Cruys et al., 2021) . A second sense of processing fluency is as an a posteriori signal reporting the effort that it actually took to process the stimulus - this might be closer to what is measured by self-reports (e.g., Forster et al., 2015) and categorization speed. Processing fluency has also been variously manipulated (Alter \& Oppenheimer, 2009).

The notion that the ease with which an object is processed influences aesthetic pleasure first emerged in the context of the mere-exposure effect (Bornstein \& D'Agostino, 1992). Bornstein suggested that repeated experience with an object facilitates its perceptual processing, resulting in a feeling of more "fluent" processing that is associated with positive affect. The "perceptual fluency" hypothesis found early empirical support (Reber et al., 1998: Winkielman \& Cacioppo, 2001) and built the basis for the idea that the relative ease of processing is the main determinant of how positively a sensed experience is evaluated (Reber et al., 2004).

A series of experiments that manipulated processing fluency, e.g., by means of priming (Albrecht \& Carbon, 2014; Reber et al., 1998), contrast (Reber et al., 1998), or stimulus presentation duration (Forster et al., 2015), has found general support for the notion that increased processing fluency leads to more positive aesthetic evaluations. In addition, processing fluency accounts can be related to several of the experimental manipulations discussed above. Specifically, symmetry and prototypicality increase processing fluency, and therefore effects of these variables on aesthetic evaluations fit generally well with processing fluency accounts. By taking into consideration the assumption that exposure facilitates processing fluency, these accounts also fit well with reports of more positive aesthetic evaluations of objects that people are exposed to more both on the short- (Anzures et al., 2009; Boothroyd et al.,2018; Cooper et al., 2006; Dotsch et al., 2016; Winkler \& Rhodes, 2005; Zajonc, 1968) and long-term (e.g., Kościński, 2012). This can be seen in terms of the rising part of the inverted U-shaped curve quantified by Montoya and colleagues (2017).

One main challenge to processing fluency accounts is the phenomenon of boredom - the falling part of Montoya and colleagues' (2017) curve. Early on, fluency proponents acknowledged that people start disliking objects that are too simple or repeated too often (Bornstein \& D'Agostino, 1992) - but these acknowledgements lack an explanation. While processing fluency theories could account for an asymptote in mere exposure effects, they cannot account for a decrease in aesthetic value judgments after a large number of exposures. Finally, the various findings regarding the relation between aesthetic value judgments and complexity are ill-fit by processing fluency theories' predictions. While, all else being equal, increasing complexity should lower processing fluency and in turn lower aesthetic value judgments, this prediction is not supported by the current evidence (e.g., Mayer and Landwehr, 2018).

Furthermore, despite the wealth of evidence, there has yet to be a more formal treatment of what actually underlies fluency. To preview, a critical component of the argument here is that fluency can be directly tied to the Helmholtzian probabilistic generative model treatment of representation that underlies analysis by synthesis (Neisser, 1967), unsupervised learning (Dayan et al., 1995: Hinton \& Sejnowski, 1999; Kingma \& Welling, 2013), predictive coding (MacKay et al., 1956: Rao \& Ballard, 1999), and, critically, various learning theories of aesthetic value (Schmidhuber, 2010). A generative model of the sensed environment enables an observer to predict her sensed experiences, or to predict future experiences given present ones. We suggest that stimuli that are 
likely or predictable under the generative model are those that are processed fluently. In later sections, we argue how adapting the generative model to make the processing of particular stimuli more fluent, i.e., to make those stimuli more likely, integrates fluency and learning theories, and addresses the challenges above.

It is particularly easy to see how good prediction might relate to fluent processing in experiences with a strong temporal component, such as music: the better the next tone in a sequence can be predicted based on the preceding tone(s), the easier it is to process, and so the greater the fluency. In general, the observer continuously predicts the next sensed input and greater fluency corresponds to a better match between this short-term prediction and the observed sensed input. In brief, one can re-phrase fluency theories as stating that sensed experiences associated with lower prediction errors are more pleasurable. However, the idea of prediction error extends beyond dynamic stimuli: analysis by synthesis (Dayan et al., 1995; MacKay et al., 1956; Neisser, 1967; Rao \& Ballard, 1999) models of the internal processing of static stimuli are also animated by prediction errors between the stimuli themselves and the models' top-down expectations of those stimuli.

\subsubsection{Learning theories}

Learning theories share the first two fundamental assumptions with processing fluency theories, namely (i) that the sensory-cognitive system of an observer is particularly suited to process objects with certain sensed features, and (ii) that there is a neural signal that indicates how well the system is suited to the features of the current sensed input. However, learning theories diverge to assume that (iii) an observer also keeps track of the development of her system's ability to process the current sensed input over time. Some add that (iv) she also keeps track of her system's ability to process the sensed environment as a whole and, to that end, takes into consideration the complete history of previously encountered sensed inputs (Schmidhuber, 2010). Learning theories then assume that (v) the relative increase (or decrease) of the system's ability to process future sensed inputs (which include any current input that persists) triggers a positive (or negative) affective response. Thus, whereas the absolute, immediate processing fluency is central to processing fluency theories, learning theories view the relative change in the observer's ability to process sensed input as the central determinant of aesthetic value.

Relative to processing fluency theories, the idea that learning about our sensed environment may be key to aesthetic value is young. The earliest formal introduction likely dates back to Jürgen Schmidhuber's "Theory of creativity, fun, and intrinsic motivation" (developed from 1990; reviewed in Schmidhuber, 2010; but see also Nake, 2012 for a review of earlier theories of aesthetic value based on the idea of information measures).

Importantly for our account, in many formal characterizations of learning, including those associated with adapting the sort of probabilistic generative models mentioned above, the signal that occasions learning in the face of a stimulus is exactly the extent to which that stimulus is unexpected - i.e., the prediction error. Indeed, the notion that the presence and resolution of prediction errors may explain aesthetic value judgements has been particularly influential over the past decade in the context of music (Gebauer et al., 2012; Koelsch et al., 2019). In the visual domain, Van de Cruys and Wagemans (Van de Cruys \& Wagemans, 2011) explicitly proposed a "prediction error account of visual art" positing that perceptual learning progress may be the source of aesthetic value. Scholars from music and vision science mainly associate a prediction error with the subjective feeling of surprise and its reduction with positive affect. 
Scientists from different fields have different predilections for the time scale over which learning progress is measured. The view from vision science emphasizes the development of processing for a single, given object. Here, pleasure is derived from a continuous decrease in prediction error for that single object, possibly culminating in an "aha"-moment (e.g., Muth and Carbon, 2013). This view pays little attention to how the adjustments made to process the current sensed input better may help or hinder the processing of future sensed inputs. Indeed, we would argue that this sort of decreasing prediction error is often better seen as an example of the online operation of a sort of analysis by synthesis algorithm we described above - tying a decrease in prediction error directly to an increase in processing fluency.

By contrast, the view inspired by computational science emphasizes the development of processing ease for the observer's entire sensed environment (Schmidhuber, 2010). This view implies that the observer possesses a model of likely future objects and that learning associated with the current sensed input aims to increase fluency of processing such future sensed experiences. Thus, stimuli are rewarded to the extent of their impact on the observer's ability to process stimuli expected to be encountered in the future. The IDyOM algorithm (Pearce, 2005) is one example in the domain of music that builds directly on this idea.

As opposed to processing fluency, there is so far no standard paradigm for testing learning accounts of aesthetic value. Indeed, the theoretical account of Van de Cruys and Wagemans, 2011 remains to be tested, as studies have yet to show a direct link between the occurrence of learning and aesthetic value judgments. However, Van Geert and Wagemans (2020) interpret results regarding the interaction between complexity and other variables, such as symmetry, in favor of a learning account. They argue that moderate felt complexity can be an indicator that the stimulus contains rich enough information for further learning while being simple enough to afford the discovery of underlying regularities. Something similar is true in the case of music, as evidenced by a recent study (Cheung et al., 2019) which shows that people rate chords low in uncertainty and high in surprise as most pleasant, which is at least consistent with a learning account. Furthermore, people spontaneously learn statistical regularities of a novel musical grammar and give higher aesthetic ratings to excerpts to which they have previously been exposed (Loui et al., 2010). However, although it is certainly clear, for instance, that people make predictions about the progression of a musical sequence, with measurable neural correlates of violations of these expectations (for a review see Koelsch et al.,2019), the relation between predictions, their violation, and subsequent updating of expectations and aesthetic value judgments in the domain of music remains to be explicitly examined.

Like processing fluency theories, learning theories also suffer from being unable to explain certain empirical findings. For one, people are readily able to make reliable aesthetic evaluations within less than one second of object exposure (Belfi et al., 2018). It is far from clear how learning progress could be correctly assessed sufficiently quickly. Second, and contrarily, self-reported aesthetic pleasure for a given stimulus remains stable between one and 30 seconds of exposure (Brielmann et al., 2017). In that sense, learning acts too fast, because it would predict that people's aesthetic valuation should change as the current stimulus is more fully understood. Third, people's tendency to revisit the same experiences over and over, such as listening to one's favorite song, is incompatible with a learning account unless one assumes that the observer will learn something new from every encounter with a given stimulus, no matter how trivial. Fourth, at least some learning theories seem to confound curiosity or interest and aesthetic value (Schmidhuber, 2010) while, at least in self-reports, people distinguish between being interested in an (art) 
object and finding it aesthetically pleasing (Schindler et al., 2017). Still, learning progress, curiosity and positive aesthetic evaluation can be linked. In the case of Mooney images, for instance, self-reported curiosity about an image increases with increasing semantic information gain (entropy in responses about the image content) and the strength of self-reported 'Aha' experiences (Van de Cruys et al.,2021). At the same time, people like a Mooney face image more the first time they see it after having had an 'Aha' moment with it (Muth \& Carbon, 2013).

\subsection{An integrative theory of aesthetic value}

We here propose a comprehensive theory of aesthetic value that integrates ideas from previous theories of aesthetic value and literature on conventional primary and secondary rewards like food and money. Our theory assumes that aesthetic value derives from the ethologically-grounded task of fashioning a sensory system that effectively processes objects that it expects to encounter, both now and in the future. This system is formed through the (neural) plasticity occasioned by the objects that are observed. The components of aesthetic value in the theory map directly onto those of conventional reinforcement learning-based computational accounts of decision making (Sutton \& Barto, 2018).

In particular, the critical focus of reinforcement learning (RL) is the integrated value of the immediate rewards that are expected to arise over the course of the long-run future. Central to RL is a temporally sophisticated form of affective (as opposed to sensory) prediction error (Sutton, 1988). This compares a current estimate of the integrated value to a previous expectation. The current estimate combines any immediate reward - the first element of the long-run future - with the expectation of the integrated value of the second and subsequent elements. Thus the net affective prediction error combines the immediate reward with the difference between new and old expectations of integrated value - i.e., the change in expectation.

In aesthetic terms, the immediate reward derives from the fluency with which the current stimulus is processed (as in fluency theories) and is quantified as the likelihood of that particular stimulus under the current generative model (see Ryali et al.,2020 for a similar operationalization that they applied to faces). The expectation of integrated reward derives from the expected likelihood of all future stimuli under the current generative model. This is a divergence between the distribution of future stimuli and the distribution implied by the current generative model, and, amongst other things is the quantity that conventionally drives probabilistic unsupervised learning (Hinton \& Sejnowski, 1999). Thus the difference between new and old expectations coming from learning is exactly the change in the fluency with which future stimuli will be processed. This learning arises from the processing of the current stimulus. The affective prediction error, which combines these two terms, is what we consider to be the aesthetic value of that stimulus. We weight the two terms to take account of the relative timescale over which the integration occurs. This theory precisely melds processing fluency theories (quantifying immediate reward) and learning theories (quantifying the benefit for the future of present observations).

In order to draw out the implications of our theory, we consider a simple version in which the probabilistic model is a multi-variate Gaussian. This version makes five simple assumptions:

1. A stimulus is represented as a vector of feature values. We represent any given stimulus at time $t$ as multi-dimensional vector $\mathbf{s}(t)$. This procedure is common practice in machine learning (e.g. Battleday et al., 2020; Hebart et al., 2020; Iigaya et al., 2020) and is the basis for how deep neural networks (DNNs) such as VGG16 (Simonyan \& Zisserman, 2015) process 
images. In theory, this feature vector can be a representation of the stimulus on any level, e.g., the raw pixel values of an image, or - at the opposite extreme - the penultimate layer of a DNN. For the purpose of introducing our model, we will here only deal with $\mathbf{s}(t)$ as an abstract representation of a small number of stimulus features (we consider this in more depth in the Discussion).

2. The observer's internal system represents these features in terms of a parameterized probabilistic model. Here, for extreme simplicity, we characterize the internal system $\mathbf{X}(t)=$ $\{\boldsymbol{\mu}(t), \Sigma\}$ as a multivariate Gaussian over $\mathbf{s}(t)$ with means $\boldsymbol{\mu}(t)$ at time $t$ and a covariance matrix $\Sigma$. For further ease, we assume that only the means are subject to change over time. Each dimension of this multivariate Gaussian represents one feature.

3. Immediate sensory reward $r(t)$ is defined as the log likelihood of a stimulus given the system state at the given time. This is an exact operationalization of processing fluency theory. Under the assumptions above, the log likelihood of a given stimulus can be calculated as the Mahalanobis distance between the stimulus feature vector $\mathbf{s}(t)$ and the system state $\mathbf{X}(t)$ :

$$
r(t)=\log [p(\mathbf{s}(t) ; \mathbf{X}(t))]=k-(\mathbf{s}(t)-\boldsymbol{\mu}(t))^{T} \Sigma^{-1}(\mathbf{s}(t)-\boldsymbol{\mu}(t)) / 2
$$

where $k$ is a constant.

4. The system state itself has a value which is equal to the average expected future sensory reward, and thus, based on the assumption above, is:

$$
V(\mathbf{X}(t) ; t)=K-K L\left(p^{\mathrm{T}}(\mathbf{s} ; t) ; p(\mathbf{s} ; \mathbf{X}(t))\right)
$$

where $K$ is a constant and $K L$ is the Kullback-Leibler divergence between the stimulus distribution $p^{\mathrm{T}}(\mathbf{s} ; t)$ at time $t$ that the subject believes will arise in the long-term future, and the distribution over those stimuli implied by the current system state. We allow for the possibility that the expected distribution can change with time, for instance as subjects become experts in a domain or realize that some stimuli are associated with other rewards (Aleem et al., 2020). However, the results we present here do not yet reflect this flexibility.

5. Experiencing a stimulus shifts the system state towards the feature values of that stimulus. Thus, $\boldsymbol{\mu}(t+1)$ is always a modification of $\boldsymbol{\mu}(t)$ such that

$$
|\boldsymbol{\mu}(t+1)-\mathbf{s}(t)|^{2} \leq|\boldsymbol{\mu}(t)-\mathbf{s}(t)|^{2}
$$

For the purpose of the current simulations, we assume that for each feature dimension $j$

$$
\mu_{j}(t+1)=\mu_{j}(t)+\alpha\left(s_{j}(t)-\mu_{j}(t)\right)
$$

where $0 \leq \alpha<1$ is a constant.

Reinforcement learning (RL; Sutton and Barto, 2018) teaches us to value the change in system state, which is here the value of the learning, as the difference between the new value of the system state, as altered by the observation $\mathbf{s}(t)$ and the original value:

$$
\Delta V(\mathbf{X}(t) ; t)=V(\mathbf{X}(t+1) ; t+1)-V(\mathbf{X}(t) ; t)
$$


Thus, changes in $\mathbf{X}(t)$ that help the processing of expected future stimuli are beneficial; changes that hurt the processing (for instance, from specializing too greatly on a particular stimulus $\mathbf{s}(t)$ that is unlikely in the future) are malign.

RL would combine the immediate reward $r(t)$ with $\Delta V(\mathbf{X}(t) ; t)$ to make the total temporal difference prediction error (see Sutton and Barto, 2018, chapter 6, and Niv and Schoenbaum, 2008 for a short review):

$$
\delta(t)=r(t)+\Delta V(\mathbf{X}(t) ; t)
$$

This quantity in turn governs choice and motivation.

Following Rutledge and colleagues (2014), we consider aesthetic value to arise from the slightly more general form

$$
A(t)=w_{0}+w^{r} r(t)+w^{V} \Delta V(\mathbf{X}(t) ; t)
$$

where $w_{0}$ is a constant bias, and $w^{r} ; w^{V} \geq 0$. We do not assume that the relative weights of immediate sensory reward and expected change in the system state's value sum to a constant. This extra generality allows us to scale the relative worth of the future to the present - ideally as an estimate of the temporally discounted number of future sensed inputs that the subject will experience. In Rutledge et al., 2014's case, immediate reward was the lottery outcome, and the prediction error term was the difference between the expected and received outcome in each trial.

Figure 1 illustrates the main components of our model for a simple, 1D example. In the following, we use the model above to simulate and account for the behavior of humans. With this, we demonstrate that the theory we propose is feasible. At the same time, because the components of our model are interpretable, these simulations provide a novel perspective on these existing empirical findings. 

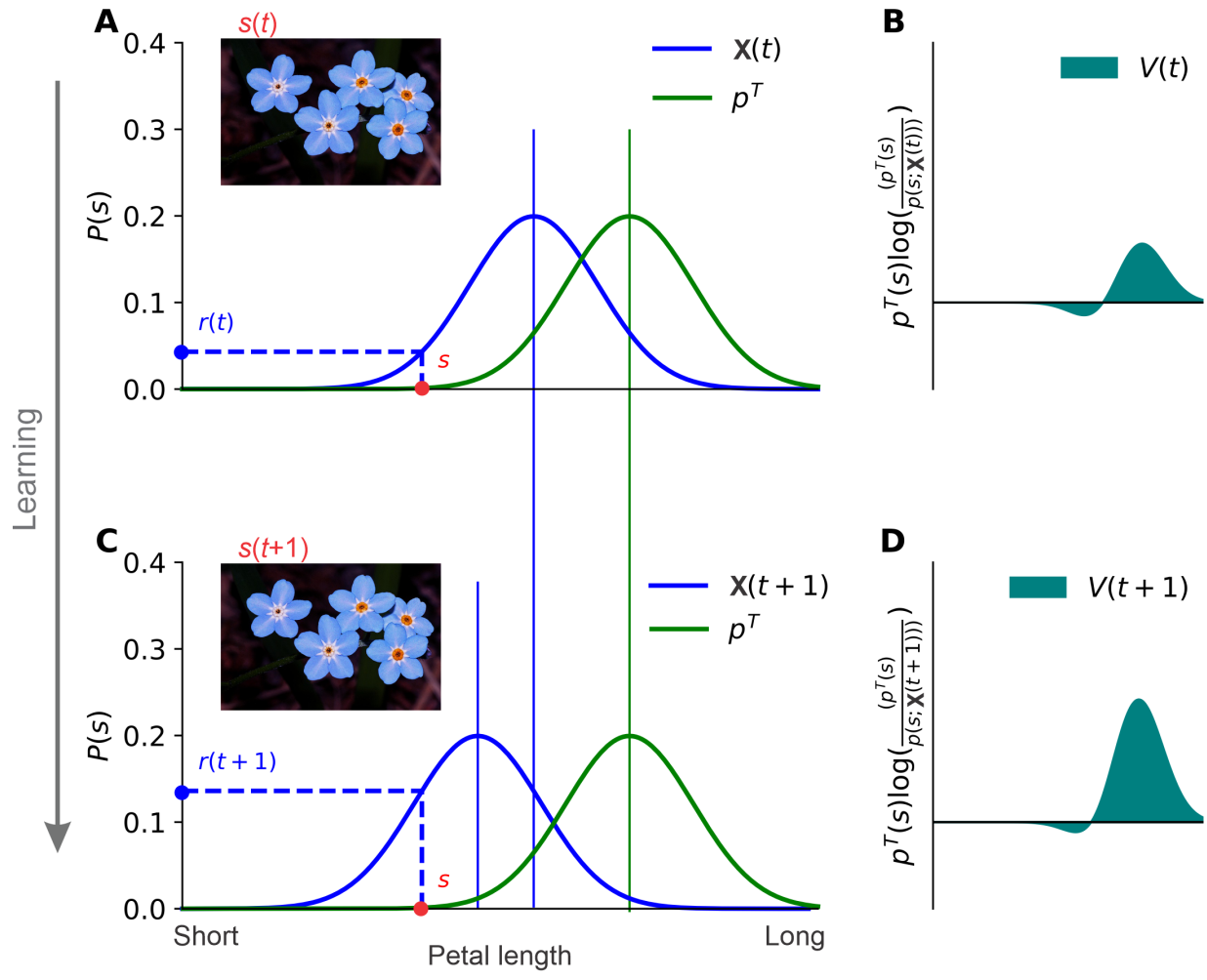

Figure 1: In this example, the only relevant feature dimension is petal length and the same stimulus (forget-me-nots) is presented at $t$ and $t+1$. Panels on the left illustrate the system state $\mathbf{X}$ (left, blue curve) and true distribution $p^{\mathrm{T}}$ (right, green curve), location of the stimulus $s$ (red dot on the x-axis), and resulting immediate sensory reward $r$ (blue dot on the y-axis). Solid vertical lines indicate the distributions' means, dashed lines illustrate the relation between $s, \mathbf{X}$, and $r$. Panels on the right illustrate the system state's value $V$ as the area under the depicted curve. The top row shows time point $t$, the bottom row $t+1$. Learning shifts the mean of the system state $\mathbf{X}(t)$ (A) to the updated mean of $\mathbf{X}(t+1)(\mathrm{C})$. As a result, $V(t)(\mathrm{B})$ changes to $V(t+1)(\mathrm{D})$. In this example, $\Delta V$ is negative because the petals of forget-me-nots are shorter than expected given the observer's system state at time $t$ and the observer expects that in the long-term future, petals will be longer than expected given her current system state's; the divergence between system state and expected true distribution therefore increases from $t$ to $t+1$. The photograph shown in this figure is licensed under a Pixabay License (free for commercial use without attribution). 


\section{Methods}

We specialize our model to simulate two paradigmatic experiments into aesthetic value judgements. The model's structural parameters are the learning rate $\alpha$; the relative weights of immediate sensory reward, $w^{r}$, and of the expected change in the system state's value, $w^{V}$; along with a constant bias $w_{0}$ to align model-predictions with the level and scale of reported results.

We consider stimuli to be $n$-dimensional, and so, for each experiment, we set the following further variable parameters: $n$ means specifying $\boldsymbol{\mu} ; n$ variances and $n(n-1) / 2$ correlations specifying $\Sigma$, as well as $n$ means, $n$ variances, and $n(n-1) / 2$ covariances specifying $p^{\mathrm{T}}$. We make the stationarity assumption that the distribution of sensed features encountered in the future, and thus $p^{\mathrm{T}}$, remains unchanged. We primarily focus on the 2D case $n=2$ (see Supplementary Material for considerations of different values for $n$ ).

For experiment 1, we fit the model to the results of a meta-analysis of mere-exposure effects (Montoya et al., 2017). For experiment 2, we fit the model to the empirical results reported by Tinio and Leder (2009) that describe aesthetic ratings as a function of stimulus complexity, symmetry, and exposure to particular levels of stimulus complexity and symmetry.

Complete code, in Python (3.8.3) running in Spyder (4.1.4), for reproducing the reported statistics and figures is available at https://github.com/aenneb/intro-aesthetic-value-model. We use the SPSQL algorithm (implemented in 'scipy.optimize') for model-fitting. We fit our model 1,000 times for each experiment, varying the random assignment of starting points across fits. We report the best fits. We generate all random variables with the numpy function random.choice() while specifying a random seed to ensure reproducibility.

\subsection{Fitting mere exposure effects}

Experiment 1 simulated a mere-exposure experiment, as in Montoya et al. (2017). We made the simplifying assumption that the covariance matrices of the system state and the expected true distribution represent the features as independent and with equal variances. For our main analysis we fix the number of features to $n=2$. Thus, we fit 10 free parameters: 2 feature values representing the stimulus, 1 variance of the system state, 2 means and 1 variance specifying the expected true distribution $p^{\mathrm{T}}$, learning rate $\alpha, w^{r}, w^{V}$, and $w_{0}$. The optimization was constrained such that $w^{r} ; w^{V} \geq 0$, and $0 \leq \alpha \leq 1$.

One experiment simulation consisted of the following steps: We first initialize the agent's system state $\mathbf{X}(0)$ with all $\boldsymbol{\mu}(0)$ values set to 0 . Next, we expose the agent to the stimulus for $m$ repeated exposures, and update $\boldsymbol{\mu}$ accordingly. We record the aesthetic value $A(m+1)$ at the ultimate exposure $m+1$. We repeat this for $1 \leq m \leq 50$ repeated exposures using the same exposure stimulus and starting with $\boldsymbol{\mu}(0)$ each time. This yields 50 aesthetic values, one per number of repeated exposures.

We fit our model to the hypothetical average ratings for each number of repeated exposures (ranging from 1 to 50) derived from the meta-analytic curve reported by Montoya and colleagues (2017). The RMSE between predicted $A(t)$ and these 50 idealized rating values serves as our measure for goodness of fit.We did not fit it to the three parameters characterizing the polynomial curve directly because polynomial fits can yield a distorted picture of the underlying data; most relevant, here, the best second-order polynomial fit to an asymptotically increasing function has an inverted-U shape. 


\subsection{Fitting the model to average liking ratings}

Experiment 2 fit our model to the data (36 average liking responses) reported by Tinio and Leder (2009). They let their participants rate stimuli of four categories: complex-symmetric (CoSy); simple-symmetric (SiSy); complex-non-symmetric (CoNs); simple-non-symmetric ( $\mathrm{SiNs}$ ). We therefore restricted our model to two feature dimensions, representing the two manipulated stimulus properties, symmetry and complexity. To manipulate exposure, Tinio and Leder introduced a familiarization procedure involving either 320 or 80 trials of exposure to stimuli of one out of the four categories (between participant manipulation).

For experiment 2 , we fit the initial means of the system state, $\boldsymbol{\mu}(0)$. For the stimuli, we fixed the feature values for simple, non-symmetric stimuli to $[0,0]$ and fit the difference in symmetry and complexity feature values that then defined the feature values for the remaining three stimulus categories. Since we fit our model to the means across 40 stimuli per stimulus category, we predicted the aesthetic value of each of these combinations of mean feature values directly, foregoing the need to assume stimulus variance.

Thus, we fit 14 free parameters for experiment 2: 2 means, 1 variance, and 1 covariance specifying the system state at $t=0,2$ means, 1 variance, and 1 covariance specifying the expected true distribution $p^{\mathrm{T}}, 2$ stimulus feature values for symmetry and complexity specifying the four stimulus categories, learning rate $\alpha, w^{r}, w^{V}$, and $w_{0}$. The optimization was constrained such that $w_{0} ; w^{r} ; w^{V} \geq 0$, and $0 \leq \alpha \leq 1$.

To obtain model predictions, we calculated the aesthetic value for each stimulus category. We simulated the familiarization procedures by exposing our model agent to an average stimulus from one of the four stimulus categories for 320 or 80 discrete time steps, one time step per familiarization trial, updating $\boldsymbol{\mu}$ accordingly, and calculating $r$ as well as $\Delta V$ for each stimulus category based on the updated value of $\boldsymbol{\mu}$. We then applied non-linear least square regression (using the scipy function optimize.nnls) to determine optimal weights $w^{r}, w^{V}$, and bias $w_{0}$. The RMSE between predicted $A(t)$ values and average ratings reported by Tinio and Leder (2009) served as our measure for goodness of fit. 


\section{Results}

In the following, we report model simulations and fits that showcase our model's ability to account for known empirical findings. We specifically focus on empirical data on judgments of pleasure and liking that reflect the scope of our theory and model of aesthetic value in the narrow sense. It is beyond the scope of a single article to provide an exhaustive treatment of all factors thought to influence aesthetic value judgments, so we here limit ourselves to a few, well-documented effects and their interaction. Since aesthetic value judgments have been most extensively studied in the visual domain, the examples we consider here are mainly visual, too (even though the metaanalysis on mere exposure effects includes data from a variety of different experiments). First, we present simulations on mere exposure effects since these have been studied intensively enough to enable meta-analytic assessment (Montoya et al., 2017). Second, we fit our model to liking ratings for simple patterns varying in symmetry and complexity - two visual object properties that have received the most attention with regard to their influence on aesthetic value judgments - that additionally examined the moderating effect of exposure (Tinio \& Leder, 2009).

\subsection{Mere exposure effect}

There is a long line of research on the so-called "mere exposure effect" (see Existing empirical findings). In an extensive meta-analysis, Montoya and colleagues (2017) summarized the findings of 81 articles providing 268 parameter estimates for polynomial fits relating the number of object exposures to the ratings they received. For this meta-analysis, all ratings were expressed as a percentage of the possible range of the rating scale, i.e., values ranged from 0 to 1 . Across all studies, Montoya and colleagues find an inverted U-shaped relationship between the number of repeated exposures and stimulus evaluations. For the 141 results regarding liking ratings, the relation between liking (which we consider a proxy for reported aesthetic value) and number of exposures is characterized by a positive slope, $b=0.0017$, and a negative quadratic term, $c=$ -0.00003 , with maximum liking ratings occurring after approximately 34 exposures.

Our model is able to capture this relationship between number of exposures and stimulus evaluation. Figure 2 A showcases the match between meta-analytic curve and predictions of the best-fitting model. The contour plot next to it illustrates the geometry of the underlying fit system state and expected true distribution. To predict the inverted U-shaped relation between number of exposures and aesthetic value, the repeatedly encountered stimulus falls inbetween the initial system state's and the expected true distribution's peaks. This results in an initial increase of both $r$ and $\Delta V$ with increasing exposure and a continuous decline in $\Delta V$ over time. As a result, the predicted aesthetic value $A$ increases as long as the increase in $r$ outweighs any detrimental effects of an increasingly negative $\Delta V$ resulting in the inverted $\mathrm{U}$-shaped function.

The study results underlying the meta-analytic results include a number of non-canonical exposure effects (Montoya, private communication). Paradigmatic examples of these can also be fit by our model; these fits are characterized by different relationships between stimulus, system state, and expected true distributions. Figures 2 B-D illustrate these alternative cases. Perhaps counter-intuitively, the fit variance of $p^{\mathrm{T}}$ was smaller than the variance of the system state for fits to all cases. 

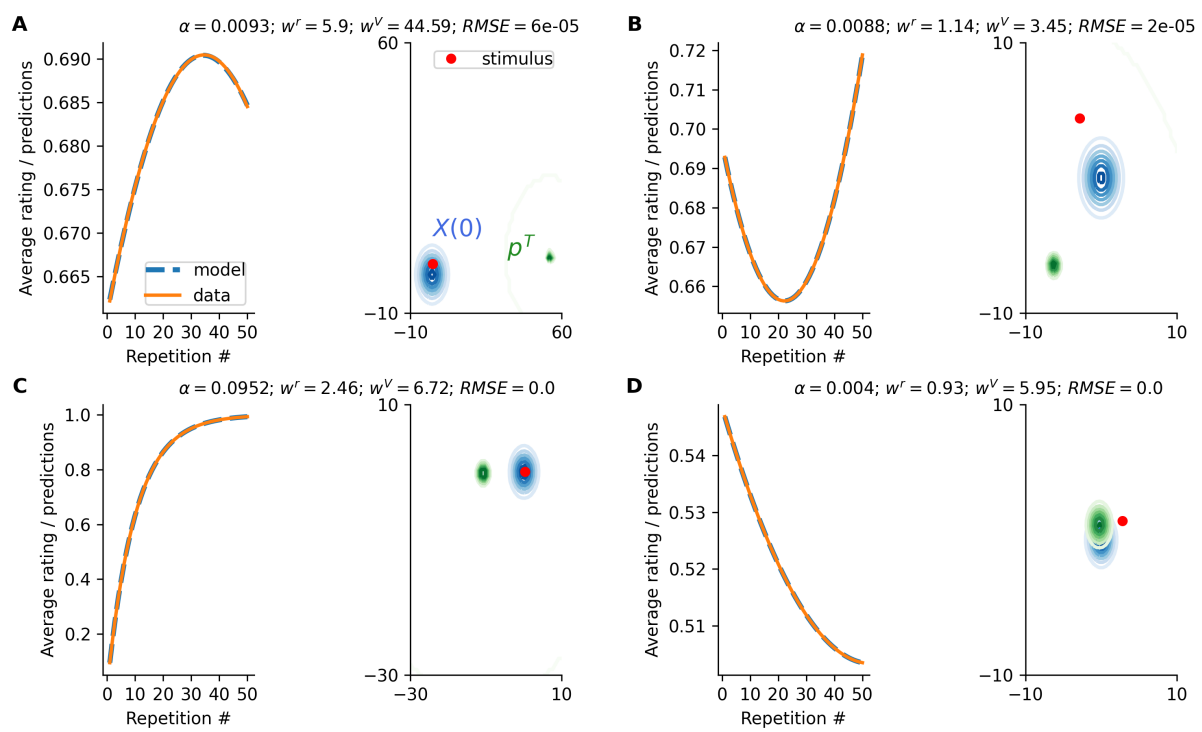

Figure 2: Model fits for different types of mere exposure effects. Headers for each panel specify parameter values and RMSE. Left side of each panel: Curve displaying the relationship between number of exposures and meta-analytic curve fits (solid orange lines) as well as model predictions (dashed blue lines). Right side of each panel: Visualization of the system state $\mathbf{X}(0)$ (blue contours), expected true distribution $p^{\mathrm{T}}$ (green contours), and stimulus (red dot) for the best-fitting model. Note that the value range is different across panels. A) Inverted-U-shaped relationship reported as the main pattern across studies by Montoya et al., 2017. B) U-shaped relationship as reported for Zajonc et al., 1974, Study 2, in Montoya et al., 2017. C) Rise to asymptote of aesthetic value with repeated exposure as observed in the raw data underlying roughly half of the invertedU-shaped curve fits shown in A). D) Decreasing relationship as reported for Gillebaart et al., 2012, Study 3, in Montoya et al., 2017. Panel titles show parameter values for the best fitting model for each of the four curves.

Of note, the relative weighting of $r$ and $\Delta V$ hardly differed between the best fits for the four different patterns; $w^{V}$ was consistently much larger than $w^{r}$. To further investigate how changes in relative weights would impact model predictions, we additionally fit modified lesioned versions of our model with either zero weight on sensory reward, or on learning, or forced equal weights on both. We fit these models to the inverted-U-shaped curve that was the main result of the metaanalysis. As shown in Figure 3, none of these alternatives fit the inverted-U-shaped curve as well as the full model. Removing either the sensory reward or the expected value component from the model resulted in a best fit that predicted an asymptotic increase in aesthetic value judgments but in an inability of the model to capture an inversion of the overall trend. 
$\mathbf{A}$
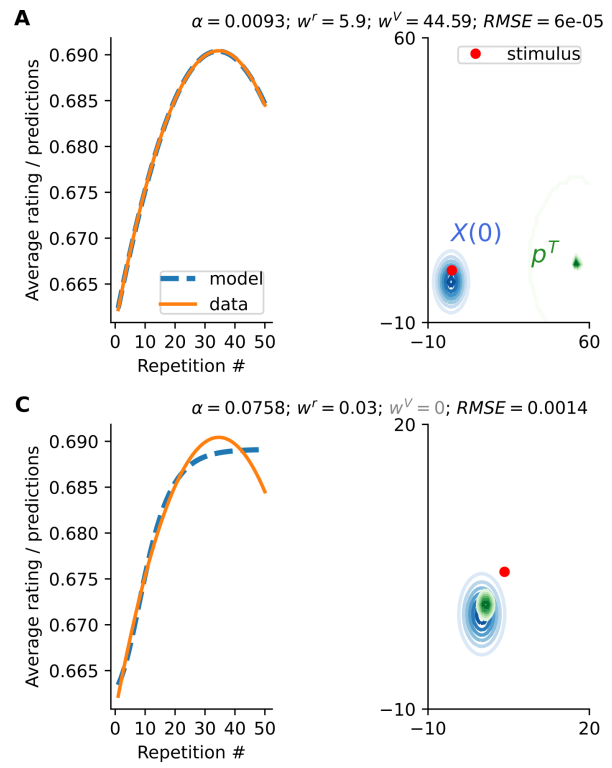
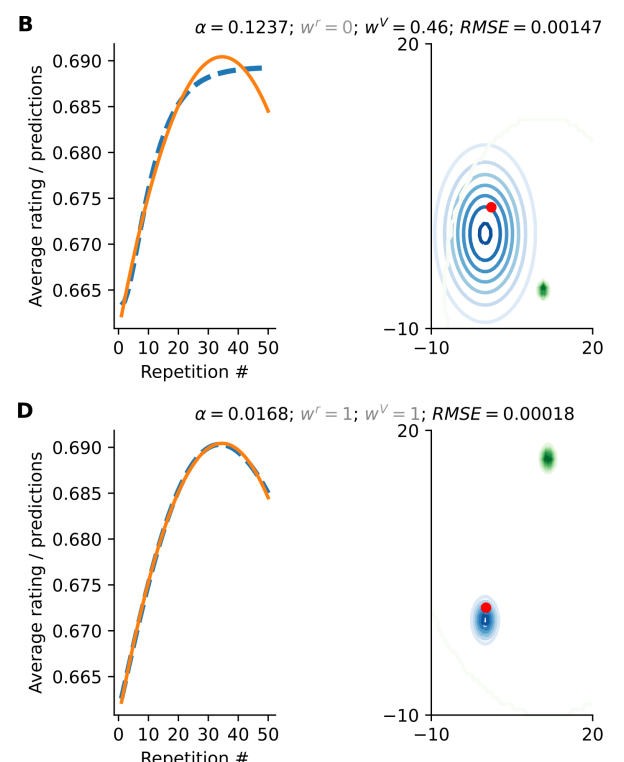

Figure 3: Best model fits for the full (A) and modified (B-D) versions of our model for mere exposure effect as reported by Montoya et al., 2017. Headers for each panel specify parameter values and RMSE; gray parameter values were fixed. Left side of each panel: Curve displaying the relationship between number of exposures and meta-analytic curve fits (solid orange lines) as well as model predictions (dashed blue lines). Right side of each panel: Visualization of the system state $\mathbf{X}(0)$ (blue contours), expected true distribution $p^{\mathrm{T}}$ (green contours), and stimulus (red dot) for the best-fitting model. A) For comparison: best model fit without modifications for reference, same as in Figure 2. B) Best fit for a model variation assuming $w^{r}=0$. C) Best fit for a model variation assuming $w^{V}=0$. D) Best fit for a model variation assuming $w^{r}=w^{V}=1$. Note that the distance between $\mathbf{X}(0)$ and $p^{\mathrm{T}}$ is smaller in $\left.\mathrm{D}\right)$ compared to $\left.\mathrm{A}\right)$ and that the learning rate is higher.

\subsection{Symmetry, complexity, and exposure}

We used the experimental results presented by Tinio and Leder (2009) as the basis for a second experiment. They report average liking ratings for four stimulus categories (complex-symmetric (CoSy); simple-symmetric (SiSy); complex-non-symmetric (CoNs); simple-non-symmetric (SiNs)) without and after exposure to either 80 or 320 stimuli of one category (between participant manipulation).

The best-fitting model well captured the overall response pattern reported by Tinio and Leder, 2009, $R M S E=0.191$ (for ratings made on a scale of 1-6; see top row of Figure 4). This best-fitting model assumes that the initial system state's mean complexity and symmetry values lie closest to the simple non-symmetric stimulus category. The means for $p^{\mathrm{T}}$, in contrast, represent stimuli as even more complex and more symmetric than the complex symmetric stimulus category. Structurally, the model that fit the data best places no weight on immediate sensory reward.

To investigate whether the non-contribution of immediate sensory reward to aesthetic value is necessary to imitate the results reported by Tinio and Leder, we also fit modified versions of our model to their data, analogous to the variations we fit to account for mere exposure effects. The middle and bottom row of Figure 4 illustrate that these modified versions of our model did 
not capture the data as well as the initial best fit. While both a model that takes only immediate sensory reward into consideration and one that assigns equal weight to $r$ and $\Delta V$ can capture the overall differences in ratings between stimulus categories, neither can account as well for the familiarization-induced changes in these ratings as the original model fits. 
A

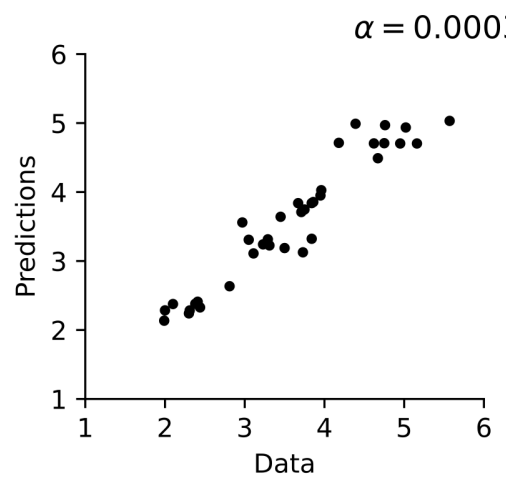

B

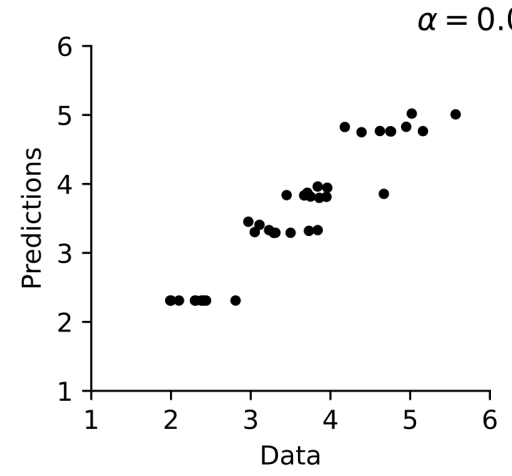

$0 \cdot w^{r}=3538 \cdot 22 \cdot w^{V}=0: R M S E$
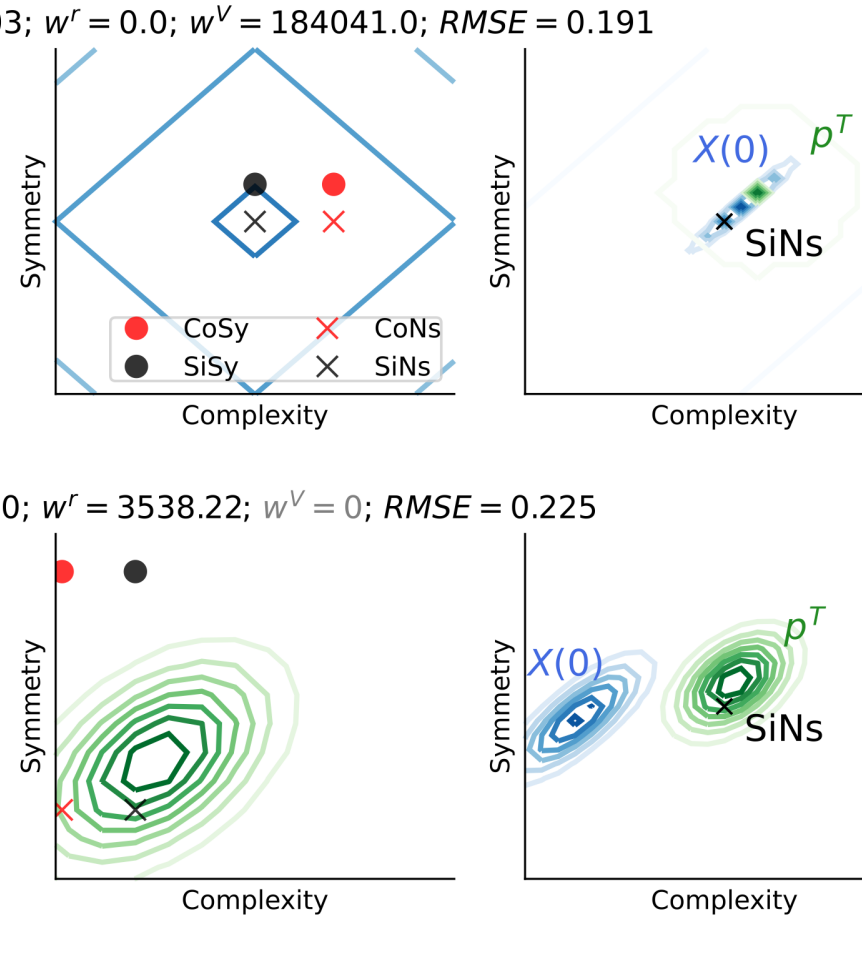

C
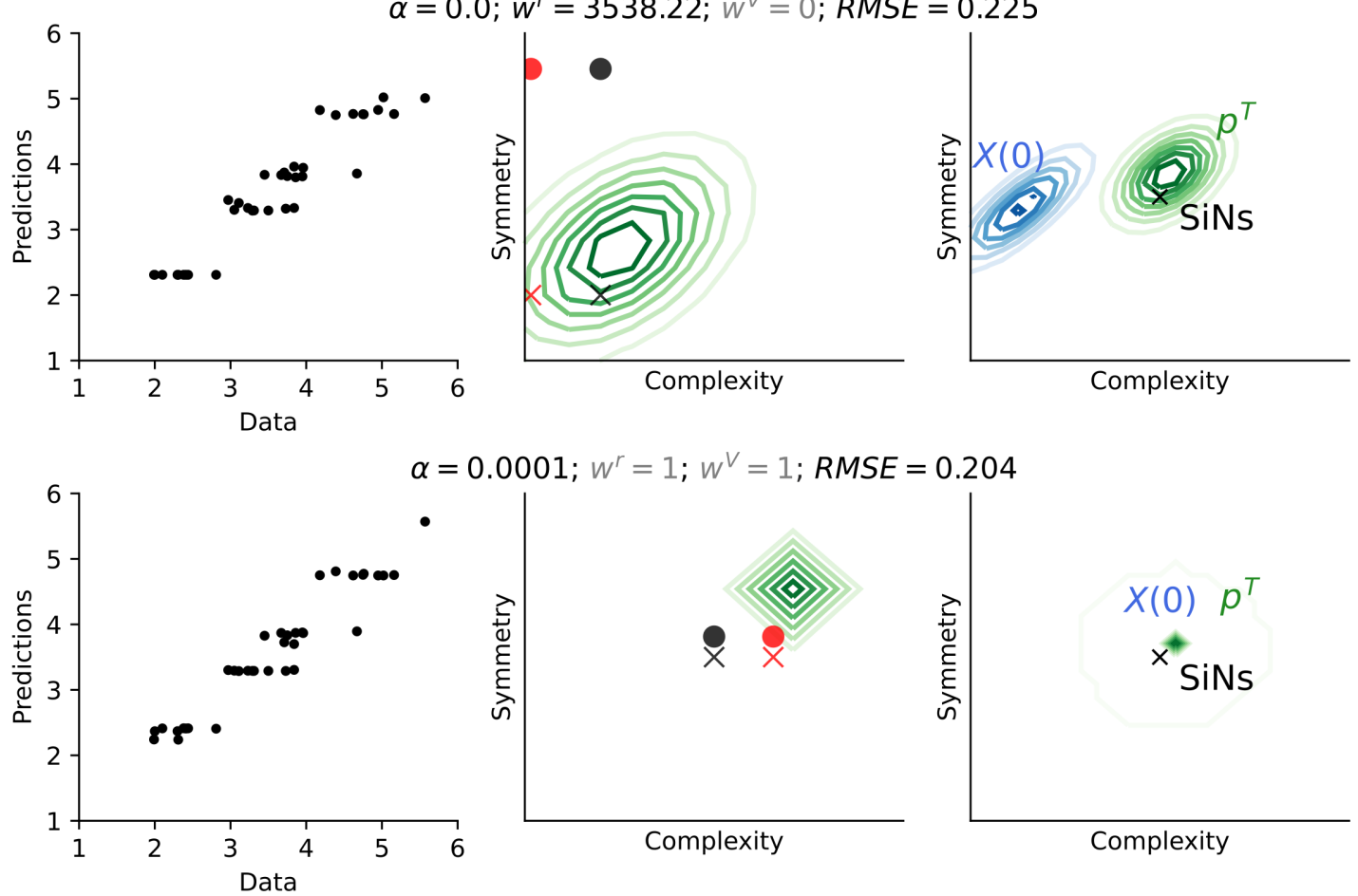

Figure 4: Model fits for the full (A) and modified (B-C) versions of our model for the average ratings reported by Tinio and Leder, 2009. Headers for each panel specify parameter values and RMSE; gray parameter values were fixed. Left side of each panel: Model predictions vs. average ratings. Each dot represents one stimulus category in one familiarization condition. Middle of each panel: Location of stimulus feature values zoomed in to a portion of the feature space. Visible contours correspond to $\mathbf{X}(0)$ (blue contours) and expected true distribution $p^{\mathrm{T}}$ (green contours). Red symbols represent complex stimuli, black ones simple stimuli; circles represent symmetric stimuli, crosses non-symmetric stimuli. Right side of each panel: Visualization of the system state $\mathbf{X}(0)$ (blue contours) and expected true distribution $p^{\mathrm{T}}$ (green contours) for the best fitting model. The black " $X$ " marks the location of simple symmetric stimulus category for reference. A) Best model fit. Note that $w^{r}=0$ for this best fit. B) Best fit for a model variation assuming $w^{V}=0$. C) Best fit for a model variation assuming $w^{r}=w^{V}=1$. Note that $\mathbf{X}(0)$ and $p^{T}$ overlap for this model fit. 


\section{Discussion}

Our theory provides a departure point for a comprehensive computational account of aesthetic value. Value is generated by two interlinked components: immediate sensory reward and a change in expected future reward; these map neatly unto the two main theories of aesthetic preference, to unsupervised learning accounts of the acquisition of generative models of sensed stimuli, and at the same time onto basic elements of reward learning models. Immediate sensory reward is closely tied to the idea of processing fluency, while the change in expected rewards is linked to the ideas of learning. In brief, our theory predicts that people find sensed experiences valuable that 1) match the makeup of the sensory-cognitive system that processes them and that 2) change the state of this system in a way that matches the properties of expected future sensed experiences. We realized this theory in a very simple model.

\subsection{Relation to existing theories}

Our theory incorporates the core ideas from processing fluency and learning accounts. The notion that objects that are easier to process have higher aesthetic value is captured as the likelihood of the object given the observer's system state, the immediate sensory reward $r$. The notion that an increase in (expected) ease of processing is aesthetically rewarding is captured as the change in the system state's value, $\Delta V$. We follow the temporal difference error from reinforcement learning in adding up these terms; quantifying their relative contribution to different aesthetic judgements. Critically, our analysis shows a deep connection between the accounts: both can be understood as relying on the notion of a generative model whose good prediction or high likelihood of sensed stimuli is positively valued. Processing fluency theories are concerned (most) with micropredictions of the current sensed input and their accuracy. Learning theories are concerned with the long-term accuracy of predictions across sensed inputs and their improvement over time. Our theory takes both short- and long-term accuracy into consideration.

Like processing fluency accounts, our theory reflects the notion that the better a sensed experience aligns with the observer's processing system, the more pleasant it is. Our theory formalizes this notion of fluency as the probability of the current sensed input given a generative model (the system state). In contrast to certain previous processing fluency accounts (Reber et al., 2004), our theory can capture (and operationalize) boredom. We demonstrated this by simulating detrimental effects of over-exposure (see figure 2). These simulations provide one illustration of our model's predictions of temporal dynamics of the evolution of aesthetic value over time.

Like learning accounts (e.g., Schmidhuber, 2010), our theory relies on the notion that the observer possesses and strives to improve a generative model of her sensed environment. In fact, we consider two generative models: the sensory system state, $\mathbf{X}$, and the expected true distribution, $p^{\mathrm{T}}$. The sensory system aims to reflect the statistical make-up of the sensed environment so that it can represent it efficiently and so predict future sensed input accurately. Learning occurs in the sense that the system state is continuously updated so as to better match the currently encountered sensed inputs.

In the current incarnation of the theory, both the generative models and the learning are extremely simple. The generative models are both unimodal, and so, in unsupervised learning terms, imply that there is only a single 'cause' of any sensed input. Central to most analysis by synthesis models of unsupervised learning is to take sensed inputs and analyze or recognize them in terms of the way that they would be synthesized or generated by the generative model. 
Here, given that the generative model only has a single cause, recognition is trivial. Furthermore, nothing about the processing of an individual stimulus, or the learning to which it leads affects this representation. Neither of these restrictions would necessarily be true given richer generative models.

In contrast to pure learning theories, our theory allows for rapid aesthetic value judgments (Belfi et al., 2018) by incorporating an element of immediate sensory reward that does not rely on computation of expected future values, and it can allow for relatively stable aesthetic value judgments over time (Brielmann et al., 2017) in cases where the current stimulus already matches the current system state.

Our theory is one of aesthetic value in the narrow sense. That is, it is concerned with aesthetic value as the basis of aesthetic evaluations of liking, pleasure, or beauty, but not with aesthetic judgments in a broader sense that would encompass a variety of different emotional responses (see Schindler et al., 2017). Our theory therefore differs from several other theories that aim to explain aesthetic valuation in this broader sense (e.g. Leder and Nadal, 2014; Pelowski et al., 2017). It is narrower than these theories in that it exclusively addresses the reward value of sensed experiences. At the same time, our theory is broader than many previous theories that focus on art (Leder \& Nadal, 2014; Pelowski et al., 2017), visual art (Van de Cruys \& Wagemans, 2011), or music (Koelsch et al.,2019) because it applies, in principle, to all sensed experiences across sensory modalities and beyond experiences labeled as 'art'.

Our theory possesses several desirable properties of previous accounts of aesthetic valuation. In particular, it is applicable to a wide range of objects of different sensory modalities (Armstrong \& Detweiler-Bedell, 2008); it is completely formalized (Birkhoff, 1933: Boselie \& Leeuwenberg, 1985: Schmidhuber, 2010); it acknowledges the fact that aesthetic values are adaptive to the environment in which they emerge (Aleem et al., 2020; though the learning process in our model differs from this previous account); it contains the notion that some sensed properties are immediately rewarding (Reber et al., 2004); and that learning about the statistics of the environment and thus improving one's sensory predictions is also rewarding (Koelsch et al., 2019; Van de Cruys \& Wagemans, 2011). Our theory and model exploit concepts that have successfully been used in reinforcement learning (RL) in order to further our understanding of aesthetic value. However, the structure of actions and state value are rather unusual in RL terms. The action in our model (namely adjusting the setting of the sensory system) is purely internal, which is rare; and the long run value of the system state depends on a statistical model of future stimuli $\left(p^{\mathrm{T}}\right)$, and yet is generating a model-free prediction error.

One more feature of our theory that is worth mentioning is its potential link to the concepts of "wanting" and "liking". The distinction between "wanting" and "liking" has previously been discussed in empirical aesthetics (Chatterjee \& Vartanian, 2016). Chatterjee and Vartanian speculated that a pure form of "liking" without "wanting" might occur when people, especially art experts, appreciate art or other objects for their aesthetic value. Our theory has the capacity to test this assumption. A very simple operationalization of "liking" would be the immediate sensory reward $r$ and "wanting" as the combination of sensory reward and expected change in the system state's value $\Delta V$ or $\delta(t)$. Thus, if Chatterjee and Vartanian (2016) are right, and we fit aesthetic responses of art experts evaluating the aesthetic value of art objects to our model, the weight of the change in the system state's value, $w^{V}$ should be approximately 0 . The average data from non-experts we fit in the current paper were not well captured by such a sensory-reward-only account (see Figures 3. 4). A fuller account of the relationship between "wanting" and "liking" might allow us some 
purchase on the complexities of the neural realization of aesthetic value, including the activation of the dopamine system (Dayan, 2021). There is also evidence that dopamine does indeed play a causal role in the experience and behavioral consequences of aesthetic value (Ferreri et al., 2019; Salimpoor et al., 2011).

\subsection{Accounting for empirical findings}

In this introductory paper, we demonstrate that a simple model based on our theory can account for the effects of exposure, symmetry, and complexity on aesthetic value judgments.

We first showed that our model can reproduce an inverted-U-shaped relation between number of exposures and aesthetic value judgments (Montoya et al., 2017). We found that this characteristic relation emerges if the (average) stimulus the agent is exposed to is moderately likely given the initial system state, and unlikely given the expected true distribution (see Figure 2 A). We thus provide a potential operationalization of the emergence of boredom after prolonged exposure that has been suggested as an amendment to processing fluency accounts of mere exposure effects (Bornstein \& D'Agostino, 1992): Objects become boring when immediate sensory reward can no longer compensate for an increasingly negative change in the value of the system state. In other words: We get bored when our increasing excellence at processing the current object can no longer compensate for our consequent decreasing competence at processing other objects we expect to encounter in the future. This can be seen as a more active form of boredom than that in learning theories, where stimuli merely lose their ability to generate positive aesthetic values when they have already been completely absorbed.

Our model can also capture the reported effects of complexity, symmetry, and exposure (Tinio \& Leder, 2009). Fitting the model, the agent's system state initially represents simple and nonsymmetrical stimuli as most likely, while the agent expects to encounter more complex and symmetrical stimuli in the future (see Figure 4 A). Aesthetic value is, in this case, computed solely based on the change in the value of the system state, placing zero weight on immediate sensory reward.

Indeed, in both experiments, and in contrast to the implication of Chatterjee and Vartanian (2016) for art experts described above, the change in the expected reward outweighed immediate reward in its importance in computing the overall value of the experience. This is in line with the results of Rutledge and colleagues (Rutledge et al., 2014) who found that moment-to-moment happiness is best predicted by a model that places higher weight on reward prediction errors than absolute current rewards. It is also consistent with an agent who expects to experience a comparatively large number of stimuli in the future. The immediate reward term might only dominate when conditions preclude or limit substantial learning. This might, for instance, be the case for faces, where a recent study has shown that the log-likelihood of a face in a representational feature space is positively correlated with its attractiveness (Ryali et al., 2020).

Much like the vast majority of previous empirical literature, we have here focused on reproducing effects on the average aesthetic judgments across objects and observers. More sophisticated versions of our model would allow it to capture individual differences in initial and evolving preferences. As an initial example of such potential differences, we here also fit our model to mere exposure effects that differ from the most prevalent pattern and were observed in individual studies (see Figure 2 B-D). The different kinds of effects were best fit with relatively stable settings of the model's structural parameters but drastically different relations between system state, stimulus, and expected true distribution. These results suggest that individual and cultural differences in 
aesthetic judgments and choices are rooted in differently shaped generative models, i.e., different past system state and expected true distribution, rather than in the weighting of immediate versus expected long-term reward or the learning processes underlying either value component. This is in line with the suggestion that, at least outside psychiatric conditions such as anhedeonia, differing past experiences, and hence likely different expected future experiences shape idiosyncrasies in aesthetic taste (see e.g., Vessel et al., 2018). This is by comparison with differences in the fundamental mechanisms underlying the computation of aesthetic value (Brielmann \& Pelli, 2018). Future experiments that provide trial-by-trial data of individual participants will have to show whether individual and cultural differences can indeed be explained by differences in generative models alone or whether structural model parameters, such as the learning rate, differ between (groups of) people, too.

The simulations we show here in most cases predicted aesthetic ratings for stimuli that are encountered repeatedly. Our model can, however, also predict the aesthetic value of objects the observer has never encountered before based on their feature values and the observer's generative models. The ability to predict the aesthetic value of novel objects is rooted in the assumption that all objects, whether novel or familiar, are represented within the same feature space. This generalized ability to predict the aesthetic value of novel objects also extends to the prediction of individual differences.

\subsection{Limitations and future directions}

In this introduction, we present basic building blocks derived from our theory. There are many directions for future development of models that are richer, and more completely specified, realizations.

First, we operated in a simplified, constrained feature space. The features we modeled here were either functionally arbitrary (Experiment 1) or abstractions of mid-level stimulus properties (symmetry, complexity, Experiment 2). However, our model could also operate on raw sensed input, such as the pixel values of an image. This would necessitate transforming raw input into an $n$-dimensional feature vector. The challenge is taken on by unsupervised learning: determining the relevant feature space, how it is learned, how it reflects the expected true distribution, and how these features are re-weighted during learning (Baldi, 2012; Barlow, 1989; Ghahramani, 2004; Hinton \& Sejnowski, 1999). Recent work in machine learning has succeeded in creating simple vector representations from images that predict human category (Battleday et al., 2020), similarity (Hebart et al., 2020), and aesthetic judgments (Iigaya et al., 2020). These feature representations can and do include high-level features. A single feature can capture semantic information, such as animacy (Hebart et al., 2020). What is more, high-level image properties, such as concreteness and valence, can be constructed from low-level image features that are extracted directly from pixel values (Iigaya et al., 2020). Based on these findings, one could imagine finding a suitable feature space for our model by exploiting feature representations of the final layers of a deep neural net (Battleday et al., 2020; Iigaya et al., 2020) or ratings of independent observers (Hebart et al., 2020). Iigaya and colleagues (2020), for instance, showed that people's liking ratings of artworks can be predicted well above chance by re-training the last three layers of VGG 16. Crucially, they also achieve far above chance predictive accuracy using PCA-compressed activation patterns from VGG 16 layers that were not re-trained on liking ratings, with higher accuracy for later layers.

Second, we made the simplifying assumption that both the system state and expected true distribution, $p^{\mathrm{T}}$, are uni-modal and that $p^{\mathrm{T}}$ is stable over time. Relaxing these assumptions could 
allow our model to capture further phenomena not considered here. Framing effects, for instance, potentially including value adaptation (Khaw et al., 2017; Polanía et al., 2019), could be understood as a change in expectations, $p^{\mathrm{T}}$, based on context. If $p^{\mathrm{T}}$ possessed multiple modes (i.e., if it was a mixture model), then the presence of a particular context, e.g., being in a museum, might trigger a switch to an expected distribution that captures the feature distribution relevant for this context, e.g., the distribution encountered in artworks. The system state might also be multimodal in the same manner. Indeed, such shifts in the shape of the underlying probability distribution of the feature space have recently been able to account for changes in attractiveness ratings of average faces in the context of gender- or race-categorization (Ryali et al., 2020). Effects of expertise could be explained by our model in an analogous manner. It seems plausible that an expert's expected true distribution of feature values contains local peaks within regions of the feature space that represent objects in her area of expertise. Changes in the expected true distribution also promise to provide a framework for understanding the impact of affective states on aesthetic responses that has drawn increasing attention in theoretical accounts (Leder \& Nadal, 2014).

Note that $p^{\mathrm{T}}$ might also benefit from plasticity - over shorter and longer terms, allowing it to adapt to local and global environments. What remains to be specified, and ultimately tested, is the time scale over which $p^{T}$ changes and how these changes (and the observer's awareness of them) influence the computation of aesthetic value. If the observer knew that her expected future distribution will likely change, for instance, then changes in the system state towards the soonto-be-outdated $p^{\mathrm{T}}$ should not weigh as heavily compared to a scenario in which she knows that $p^{\mathrm{T}}$ will remain relatively stable. More speculatively, the observer might have some expectations about the nature of this change to $p^{\mathrm{T}}$, and determine a progressive diet of stimuli that will lead quickly and effectively to a matched system state. This would be an example of (self-)shaping - a term borrowed from the instrumental conditioning literature as to the gradual acquisition of competence in complex tasks (Krueger \& Dayan, 2009; Skinner, 1975). Unfortunately, we know from research on affective forecasting that people often perform poorly when asked to predict what their affective responses (Gilbert \& Wilson, 2007), including aesthetic preferences (Quoidbach et al., 2013) will be in the future. It might be that people are reluctant to countenance such changes, and so will not optimize the stimuli they seek to observe, or that people will fail to take such predicted changes in $p^{\mathrm{T}}$ fully into account in making their predictions.

Third, many other intricacies of our model remain to be specified. How exactly the value of the current and expected system state is computed, for instance, is far from settled. We used the Kullback-Leibler divergence, since this exactly captures the average fluency. However, this is based on the implicit assumption that both the system state and $p^{\mathrm{T}}$ will subsequently remain fixed. This assumption is likely incorrect. Finding an alternative way to calculate the value of the current and expected system state for now remains an open issue for future implementations of our model.

Fourth, we focused on examples drawn mostly from vision and audition. Within the domain of vision, we focused on three rather specific examples of well-documented effects, i.e., exposure, symmetry, and complexity. Future work should extend the application of our theory to a variety of different effects. The widespread appearance of inverted-U-shaped relations between stimulus features and aesthetic judgments lends itself as a potentially fruitful starting point for such extensions because of the way they can be explained by the feature values' location relative to the means of system state and expected future distribution. Low-points of the inverted-U-shape should correspond to feature values that drive the system state's mean away from the centers of 
$p^{\mathrm{T}}$ and the current system state, whereas its peak should correspond to feature values that are both close to the current system state's mean and shift it towards the center of $p^{\mathrm{T}}$. Our focus on vision and audition reflects the current bias of research on aesthetic valuation and judgments but not the scope of our theory. In principle, our theory can be applied to any sensory modality, including olfaction, taste, and haptics. As long as one can represent the stimulus in terms of several features and one can generate a probability distribution for the values of these features, our model can, theoretically, be applied to any kind of stimulus. That being said, other modalities do pose additional challenges such as the influence of satiation when it comes to taste.

Fifth, our theory assumes that the system state continuously changes - an assumption that some might want to challenge. Why should one not selectively 'switch off' learning in cases where further adjustments of the system state become detrimental to its expected future value (cases which we consider instances of boredom)? We suggest that there is no need for an additional mechanism that suspends learning. Instead, the affective-behavioral implications of boredom in themselves - quitting the current environment and seeking out or exploring for a different one so as to avoid the negative affect of being bored - provide a solution to the problem of detrimental learning. In a constantly changing environment it may even represent a better solution than ceasing to learn. When a (local) maximum in the value of the system state does not represent a truly optimal solution, it is more beneficial to continuously learn and actively seek out new information than to remain stationary and cease adaptation once a temporarily optimal solution has been found.

Sixth, a related feature of our account that might seem puzzling to some readers is the presence of two generative models, held simultaneously. Why should the agent possess a system state that is different from the expected true distribution? One consideration is that our theory ultimately aims to reflect the behavior and judgments of people who live in a constantly changing sensed environment that has no certain, known future state. In such an environment we suggest that it makes sense to maintain both a model of the current sensed environment, $\mathbf{X}$, and a separate model of the probable future environment, $p^{\mathrm{T}}$. Like this, the agent can become better at predicting her current environment over time - the more so, the longer she happens to find herself in it. This might be particularly beneficial in cases where the objective true distribution has changed but the agent has not (yet) updated $p^{\mathrm{T}}$. At the same time, keeping track of the development of the distance between the current system state and the expected true distribution $(\Delta V)$ remains important as an indicator for how far this locally better-adjusted system state is removed from the makeup of currently expected future environments.

Imagine, for instance, that you live in the mountains but temporarily find yourself at the beach. Adjusting your sensory system to the new environment is beneficial for navigating your new, flat environment even though your long-term expectations may still reflect decades of experience with hilly terrain. Depending on how long you stay at the beach, it may be beneficial to keep your long-term expectations the same (you went on a day trip), change them (you moved permanently) or create a separate long-term expectation, a separate mode for $p^{\mathrm{T}}$ (you bought a weekend home). This example illustrates how maintaining a separate system state and expected true distribution allows flexible adjustments based on different patterns of changing environments. It also illustrates the usefulness of further developments of our theory that include non-stationary and multi-modal instances of the expected true distribution.

Finally, in the form presented here, our theory is a theory of aesthetic value. It is, however, designed to be adaptable to become a theory of aesthetic choice. Previous treatments of aesthetic 
valuation (e.g., Leder and Nadal, 2014) make qualitative predictions about the variables that influence aesthetic judgments but they do not predict individual choices between concrete options. Our theory can be extended to do so because it computes a numerical aesthetic value for individual objects. By adding a decision-rule that compares the current or expected aesthetic value of one option against another or a set threshold, our theory can easily be extended to predict aesthetic choice. In this context of aesthetic choice, the explore/exploit dilemma (Cohen et al., 2007) will be particularly relevant: How long should I continue to engage with a particular sensed experience, such as listening to a song? And when is is time to switch to a different one?

\subsection{Conclusion}

The aesthetic value of sensed experiences shapes many of our everyday decisions. Here, we introduced a theory of aesthetic value based on the idea that the observer strives to adapt her sensory system such as to effectively processes objects now and in the future. In this sense, sensed experiences can be valuable in two ways. First, experiences are valuable to the extent that they already match the current system state, allowing immediate efficient processing. Second, experiences are valuable to the extent that they change the system state in such a way that expected future experiences are processed more efficiently. The simple model of our theory presented in this paper can account for empirical data and sheds new insight on how immediate processing fluency and learning jointly influence aesthetic value judgments. 


\section{Acknowledgments}

We thank Maria Pombo for feedback on an earlier version of this manuscript. We thank Mihály Bányai, Amy Belfi, Gabriele Bellucci, Noémi Éltető, Susanne Haridi, Denis Pelli, Lion Schulz, Miriam Spering, and Edward Vessel for helpful comments. We thank R. Matthew Montoya for sharing the data underlying the meta-analysis of the mere exposure effect and his insights into different patterns of mere exposure effects. We thank Shervin Safavi for reviewing the code associated with this paper and its methods section. This work was supported by the Max Planck Society, the Alexander von Humboldt foundation, and the Deutsche Forschungsgemeinschaft (DFG, German Research Foundation). 


\section{References}

Albrecht, S., \& Carbon, C.-C. (2014). The fluency amplification model: Fluent stimuli show more intense but not evidently more positive evaluations. Acta Psychologica, 148, 195-203. https: //doi.org/10.1016/j.actpsy.2014.02.002

Aleem, H., Correa-Herran, I., \& Grzywacz, N. M. (2020). A theoretical framework for how we learn aesthetic values. Frontiers in Human Neuroscience, 14, 345. https:/ / doi.org/10.3389/ fnhum.2020.00345

Alter, A. L., \& Oppenheimer, D. M. (2009). Uniting the tribes of fluency to form a metacognitive nation. Personality and Social Psychology Review, 13(3), 219-235. https://doi.org/10.1177/ 1088868309341564

Anglada-Tort, M., \& Skov, M. (2020). What counts as aesthetics in science? a bibliometric analysis and visualization of the scientific literature from 1970 to 2018. Psychology of aesthetics, creativity, and the arts. https://doi.org/10.1037/aca0000350

Anglada-Tort, M., Steffens, J., \& Müllensiefen, D. (2019). Names and titles matter: The impact of linguistic fluency and the affect heuristic on aesthetic and value judgements of music. Psychology of aesthetics, creativity, and the arts, 13(3), 277-292. https: / / doi.org / 10.1037/ aca0000172

Anzures, G., Mondloch, C. J., \& Lackner, C. (2009). Face adaptation and attractiveness aftereffects in 8-year-olds and adults. Child Development, 80(1), 178-191. https://doi.org/10.1111/j. 1467-8624.2008.01253.x

Armstrong, T., \& Detweiler-Bedell, B. (2008). Beauty as an emotion: The exhilarating prospect of mastering a challenging world. Review of General Psychology, 12(4), 305-329. https: / / doi. org/10.1037/a0012558

Baldi, P. (2012). Autoencoders, unsupervised learning, and deep architectures. Proceedings of ICML workshop on unsupervised and transfer learning, 37-49.

Barlow, H. B. (1989). Unsupervised learning. Neural computation, 1(3), 295-311.

Battleday, R. M., Peterson, J. C., \& Griffiths, T. L. (2020). Capturing human categorization of natural images by combining deep networks and cognitive models. Nature Communications, 11(1), 5418. https://doi.org/10.1038/s41467-020-18946-z

Belfi, A. M., Kasdan, A., Rowland, J., Vessel, E. A., Starr, G. G., \& Poeppel, D. (2018). Rapid timing of musical aesthetic judgments. Journal of Experimental Psychology: General, 147(10), 15311543. https://doi.org/10.1037/xge0000474

Berlyne, D. E. (1970). Novelty, complexity, and hedonic value. Perception E Psychophysics, 8(5A), 279-286.

Berridge, K. C., Robinson, T. E., \& Aldridge, J. W. (2009). Dissecting components of reward: 'liking', 'wanting', and learning. Current Opinion in Pharmacology, 9(1), 65-73. https://doi.org/10. 1016/j.coph.2008.12.014

Birkhoff, G. D. (1933). Aesthetic measure. Cambridge, Mass.

Boothroyd, L., Pollet, T. V., Evans, E., Yi, Q., \& Tovee, M. (2018). Adaptation-like effects in body weight attractiveness are not simply norm based. https://doi.org/10.31234/osf.io/gsnkf

Bornstein, R. F., \& D'Agostino, P. R. (1992). Stimulus recognition and the mere exposure effect. Journal of Personality and Social Psychology, 63(4), 545-552. https:// doi.org/10.1037/00223514.63.4.545 
Boselie, F., \& Leeuwenberg, E. (1985). Birkhoff revisited: Beauty as a function of effect and means. The American Journal of Psychology, 98(1), 1-39. Retrieved November 9, 2020, from https: //www.ncbi.nlm.nih.gov/pubmed/4003614

Brattico, E., Pallesen, K. J., Varyagina, O., Bailey, C., Anourova, I., Järvenpää, M., Eerola, T., \& Tervaniemi, M. (2009). Neural discrimination of nonprototypical chords in music experts and laymen: An MEG study. Journal of Cognitive Neuroscience, 21(11), 2230-2244. https:// doi.org/10.1162/jocn.2008.21144

Brielmann, A. A., \& Pelli, D. G. (2018). Aesthetics. Current Biology, 28(16), R859-R863. https:/ /doi. org/10.1016/j.cub.2018.06.004

Brielmann, A. A., Vale, L., \& Pelli, D. G. (2017). Beauty at a glance: The feeling of beauty and the amplitude of pleasure are independent of stimulus duration. Journal of Vision, 17(14), 9. https://doi.org/10.1167/17.14.9

Brown, S., Gao, X., Tisdelle, L., Eickhoff, S. B., \& Liotti, M. (2011). Naturalizing aesthetics: Brain areas for aesthetic appraisal across sensory modalities. Neuroimage, 58(1), 250-258. https: //doi.org/10.1016/j.neuroimage.2011.06.012

Chatterjee, A., \& Vartanian, O. (2016). Neuroscience of aesthetics. Annals of the New York Academy of Sciences, 1369(1), 172-194. https://doi.org/10.1111/nyas.13035

Cheung, V. K. M., Harrison, P. M. C., Meyer, L., Pearce, 3. T., Haynes, J.-D., \& Koelsch, S. (2019). Uncertainty and surprise jointly predict musical pleasure and amygdala, hippocampus, and auditory cortex activity. Current Biology, 29(23), 4084-4092.e4. https: / / doi.org / 10. 1016/j.cub.2019.09.067

Christensen, B. T., Ball, L. J., \& Reber, R. (2020). Perceptual fluency effects in judgments of creativity and beauty: Creative objects are perceived fluently yet they are visually complex. Journal of Cognitive Psychology, 32(1), 45-66. https://doi.org/10.1080/20445911.2019.1689986

Cohen, J. D., McClure, S. M., \& Yu, A. J. (2007). Should i stay or should i go? how the human brain manages the trade-off between exploitation and exploration. Philosophical Transactions of the Royal Society of London. Series B, Biological Sciences, 362(1481), 933-942. https://doi.org/ $10.1098 / \mathrm{rstb} .2007 .2098$

Cooper, P. A., Geldart, S. S., Mondloch, C. J., \& Maurer, D. (2006). Developmental changes in perceptions of attractiveness: A role of experience? Developmental Science, 9(5), 530-543. https://doi.org/10.1111/j.1467-7687.2006.00520.x

Corradi, G., Chuquichambi, E. G., Barrada, J. R., Clemente, A., \& Nadal, M. (2020). A new conception of visual aesthetic sensitivity. British Journal of Psychology, 111(4), 630-658. https: //doi.org/10.1111/bjop.12427

Dayan, P. (2021). When will's wont wants wanting. Behavioral and Brain Sciences, 44.

Dayan, P., Hinton, G. E., Neal, R. M., \& Zemel, R. S. (1995). The Helmholtz machine. Neural computation, 7(5), 889-904.

Dotsch, R., Hassin, R. R., \& Todorov, A. (2016). Statistical learning shapes face evaluation. Nature Human Behaviour, 1(1), 0001. https://doi.org/10.1038/s41562-016-0001

Dubey, R., Ho, M. K., Mehta, H., \& Griffiths, T. (2021). Aha! moments correspond to meta-cognitive prediction errors. https://doi.org/10.31234/osf.io/c5v42

Fechner, G. T. (1876). Vorschule der aesthetik (Vol. 1). Breitkopf \& Härtel.

Ferreri, L., Mas-Herrero, E., Zatorre, R. J., Ripollés, P., Gomez-Andres, A., Alicart, H., Olivé, G., Marco-Pallarés, J., Antonijoan, R. M., Valle, M., Riba, J., \& Rodriguez-Fornells, A. (2019). Dopamine modulates the reward experiences elicited by music. Proceedings of the National 
Academy of Sciences of the United States of America, 116(9), 3793-3798. https:/ / doi.org/10. 1073/pnas.1811878116

Forster, M., Gerger, G., \& Leder, H. (2015). Everything's relative? relative differences in processing fluency and the effects on liking. Plos One, 10(8), e0135944. https: / / doi.org / 10.1371/ journal.pone.0135944

Gartus, A., Völker, M., \& Leder, H. (2020). What experts appreciate in patterns: Art expertise modulates preference for asymmetric and face-like patterns. Symmetry: Culture and Science, 12(5), 707. https://doi.org/10.3390/sym12050707

Geana, A., Wilson, R. C., Daw, N., \& Cohen, J. (2016). Boredom, information-seeking and exploration. In A. Papafragou, D. Grodner, D. Mirman, \& J. Trueswell (Eds.), Proceedings of the 38th annual conference of the cognitive science society (pp. 1751-1756). Cognitive Science Spciety.

Gebauer, L., Kringelbach, M. L., \& Vuust, P. (2012). Ever-changing cycles of musical pleasure: The role of dopamine and anticipation. Psychomusicology: Music, Mind, and Brain, 22(2), 152167. https://doi.org/10.1037/a0031126

Gerger, G., \& Leder, H. (2015). Titles change the esthetic appreciations of paintings. Frontiers in Human Neuroscience, 9, 464. https:/ /doi.org/10.3389/fnhum.2015.00464

Ghahramani, Z. (2004). Unsupervised learning. In O. Bousquet, U. von Luxburg, \& G. Rätsch (Eds.), Advanced lectures on machine learning: Ml summer schools 2003, canberra, australia, february 2 - 14, 2003, tübingen, germany, august 4 - 16, 2003, revised lectures (pp. 72-112). Springer Berlin Heidelberg. https://doi.org/10.1007/978-3-540-28650-9_5

Gilbert, D. T., \& Wilson, T. D. (2007). Prospection: Experiencing the future. Science, 317(5843), 13511354. https://doi.org/10.1126/science.1144161

Gillebaart, M., Förster, J., \& Rotteveel, M. (2012). Mere exposure revisited: The influence of growth versus security cues on evaluations of novel and familiar stimuli. Journal of Experimental Psychology: General, 141(4), 699.

Halberstadt, J., \& Rhodes, G. (2003). It's not just average faces that are attractive: Computermanipulated averageness makes birds, fish, and automobiles attractive. Psychonomic Bulletin $\mathcal{E}$ Review, 10(1), 149-156. https://doi.org/10.3758/bf03196479

Hebart, 3. N., Zheng, C., Pereira, F., \& Baker, C. I. (2020). Revealing the multidimensional mental representations of natural objects underlying human similarity judgments. https: / / doi. org/10.31234/osf.io/7wrgh

Hinton, G. E., \& Sejnowski, T. J. (Eds.). (1999). Unsupervised learning: Foundations of neural computation. MIT Press.

Höfel, L., \& Jacobsen, T. (2007). Electrophysiological indices of processing aesthetics: Spontaneous or intentional processes? International Journal of Psychophysiology, 65(1), 20-31. https://doi. org/10.1016/j.ijpsycho.2007.02.007

Iigaya, K., Yi, S., Wahle, I. A., Tanwisuth, K., \& O’Doherty, J. P. (2020). Aesthetic preference for art emerges from a weighted integration over hierarchically structured visual features in the brain. BioRxiv. https://doi.org/10.1101/2020.02.09.940353

Jacobsen, T., \& Höfel, L. (2002). Aesthetic judgments of novel graphic patterns: Analyses of individual judgments. Perceptual and Motor Skills, 95(3), 755-766. https: / / doi.org / 10.2466/ pms.2002.95.3.755 
Jacobsen, T., Schubotz, R. I., Höfel, L., \& Cramon, D. Y. V. (2006). Brain correlates of aesthetic judgment of beauty. Neuroimage, 29(1), 276-285. https:// doi.org/10.1016/j.neuroimage. 2005.07.010

Kahneman, D. (2011). Thinking, fast and slow. Macmillan.

Khaw, M. W., Glimcher, P. W., \& Louie, K. (2017). Normalized value coding explains dynamic adaptation in the human valuation process. Proceedings of the National Academy of Sciences of the United States of America, 114(48), 12696-12701. https: / / doi.org / 10.1073 / pnas. 1715293114

Kingma, D. P., \& Welling, M. (2013). Auto-encoding variational bayes. arXiv preprint arXiv:1312.6114.

Kirk, U., Skov, M., Hulme, O., Christensen, M. S., \& Zeki, S. (2009). Modulation of aesthetic value by semantic context: An fMRI study. Neuroimage, 44(3), 1125-1132. https: / / doi.org/10. 1016/j.neuroimage.2008.10.009

Koelsch, S., Vuust, P., \& Friston, K. (2019). Predictive processes and the peculiar case of music. Trends in Cognitive Sciences, 23(1), 63-77. https://doi.org/10.1016/j.tics.2018.10.006

Kościński, K. (2012). Mere visual experience impacts preference for body shape: Evidence from male competitive swimmers. Evolution and Human Behavior, 33(2), 137-146. https: / / doi. org/10.1016/j.evolhumbehav.2011.08.003

Krueger, K. A., \& Dayan, P. (2009). Flexible shaping: How learning in small steps helps. Cognition, 110(3), 380-394.

Kühn, S., \& Gallinat, J. (2012). The neural correlates of subjective pleasantness. Neuroimage, 61(1), 289-294. https://doi.org/10.1016/j.neuroimage.2012.02.065

Leder, H., Carbon, C.-C., \& Ripsas, A.-L. (2006). Entitling art: Influence of title information on understanding and appreciation of paintings. Acta Psychologica, 121(2), 176-198. https:// doi.org/10.1016/j.actpsy.2005.08.005

Leder, H., \& Nadal, 3. (2014). Ten years of a model of aesthetic appreciation and aesthetic judgments : The aesthetic episode - developments and challenges in empirical aesthetics. British Journal of Psychology, 105(4), 443-464. https://doi.org/10.1111/bjop.12084

Leder, H., Tinio, P. P. L., Brieber, D., Kröner, T., Jacobsen, T., \& Rosenberg, R. (2018). Symmetry is not a universal law of beauty. Empirical Studies of the Arts, 37(1), 027623741877794. https: //doi.org/10.1177/0276237418777941

Loui, P., Wessel, D. L., \& Hudson Kam, C. L. (2010). Humans rapidly learn grammatical structure in a new musical scale. Music perception, 27(5), 377-388. https://doi.org/10.1525/mp.2010. 27.5.377

Lyssenko, N., Redies, C., \& Hayn-Leichsenring, G. U. (2016). Evaluating abstract art: Relation between term usage, subjective ratings, image properties and personality traits. Frontiers in Psychology, 7, 973. https://doi.org/10.3389/fpsyg.2016.00973

MacKay, D. M., ASHBY, W. R., CULBERTSON, J. T., DAVIS, M. D., KLEENE, S. C., LEEUW, K. D., KAY, D. M. M., CARTHY, J. M., MINSKY, M. L., MOORE, E. F., SHANNON, C. E., SHAPIRO, N., UTTLEY, A. M., \& NEUMANN, J. V. (1956). The epistemological problem for automata. Automata studies. (am-34) (pp. 235-252). Princeton University Press. http: //www.jstor.org/stable/j.ctt1bgzb3s.14

Marin, M. M., Lampatz, A., Wandl, M., \& Leder, H. (2016). Berlyne revisited: Evidence for the multifaceted nature of hedonic tone in the appreciation of paintings and music. Frontiers in Human Neuroscience, 10, 536. https:/ / doi.org/10.3389/fnhum.2016.00536 
Martindale, C., \& Moore, K. (1988). Priming, prototypicality, and preference. Journal of Experimental Psychology: Human Perception and Performance, 14(4), 661-670. https: / / doi.org / 10.1037/ 0096-1523.14.4.661

Martindale, C., Moore, K., \& West, A. (1988). Relationship of preference judgments to typicality, novelty, and mere exposure. Empirical Studies of the Arts, 6(1), 79-96. https:// doi.org/10. 2190/\{MCAJ $\}-\{0 G Q T\}-\{$ DJTL $\}-\{$ LNQD $\}$

Mas-Herrero, E., Maini, L., Sescousse, G., \& Zatorre, R. J. (2021). Common and distinct neural correlates of music and food-induced pleasure: A coordinate-based meta-analysis of neuroimaging studies. Neuroscience and Biobehavioral Reviews, 123, 61-71. https://doi.org/10. 1016/j.neubiorev.2020.12.008

Mayer, S., \& Landwehr, J. R. (2018). Quantifying visual aesthetics based on processing fluency theory: Four algorithmic measures for antecedents of aesthetic preferences. Psychology of aesthetics, creativity, and the arts, 12(4), 399-431. https://doi.org/10.1037/aca0000187

McClure, S. M., Li, J., Tomlin, D., Cypert, K. S., Montague, L. M., \& Montague, P. R. (2004). Neural correlates of behavioral preference for culturally familiar drinks. Neuron, 44(2), 379-387. https://doi.org/10.1016/j.neuron.2004.09.019

Mealey, L., Bridgstock, R., \& Townsend, G. C. (1999). Symmetry and perceived facial attractiveness: A monozygotic co-twin comparison. Journal of Personality and Social Psychology, 76(1), 151-158. Retrieved November 9, 2020, from https:/ / www.ncbi.nlm.nih.gov / pubmed/ 9972560

Millis, K. (2001). Making meaning brings pleasure: The influence of titles on aesthetic experiences. Emotion, 1(3), 320-329. https://doi.org/10.1037//1528-3542.1.3.320

Montoya, R. M., Horton, R. S., Vevea, J. L., Citkowicz, 3., \& Lauber, E. A. (2017). A re-examination of the mere exposure effect: The influence of repeated exposure on recognition, familiarity, and liking. Psychological Bulletin, 143(5), 459-498. https://doi.org/10.1037/bul0000085

Mullennix, J. W., \& Robinet, J. (2018). Art expertise and the processing of titled abstract art. Perception, 47(4), 359-378. https://doi.org/10.1177/0301006617752314

Muñoz-Reyes, J. A., Iglesias-Julios, M., Pita, M., \& Turiegano, E. (2015). Facial features: What women perceive as attractive and what men consider attractive. Plos One, 10(7), e0132979. https://doi.org/10.1371/journal.pone.0132979

Muth, C., \& Carbon, C.-C. (2013). The aesthetic aha: On the pleasure of having insights into gestalt. Acta Psychologica, 144(1), 25-30. https://doi.org/10.1016/j.actpsy.2013.05.001

Nadal, M., Munar, E., Marty, G., \& Cela-Conde, C. J. (2010). Visual complexity and beauty appreciation: Explaining the divergence of results. Empirical Studies of the Arts, 28(2), 173-191. https://doi.org/10.2190/\{EM\}.28.2.d

Nake, F. (2012). Information aesthetics: An heroic experiment. Journal of Mathematics and the Arts, 6(2-3), 65-75. https://doi.org/10.1080/17513472.2012.679458

Neisser, U. (1967). Cognitive psychology. Appleton-Century-Crofts. https: / / works.swarthmore. edu/alum-books/2806/

Newman, G. E., \& Bloom, P. (2012). Art and authenticity: The importance of originals in judgments of value. Journal of Experimental Psychology: General, 141(3), 558-569. https:/ / doi.org/10. $1037 / \mathrm{a} 0026035$

Niv, Y., \& Schoenbaum, G. (2008). Dialogues on prediction errors. Trends in cognitive sciences, 12(7), 265-272. 
Palmer, S. E., Schloss, K. B., \& Sammartino, J. (2013). Visual aesthetics and human preference. Annual review of psychology, 64, 77-107. https://doi.org/10.1146/annurev-psych-120710100504

Pearce, 3. T. (2005). The construction and evaluation of statistical models of melodic structure in music perception and composition (Doctoral dissertation). City University London.

Pelowski, M., Markey, P. S., Forster, M., Gerger, G., \& Leder, H. (2017). What do we actually hope to accomplish by modeling art experience?: Reply to comments on "move me, astonish me... delight my eyes and brain: The vienna integrated model of top-down and bottom-up processes in art perception (VIMAP) and corresponding affective, evaluative, and neurophysiological correlates". Physics of life reviews, 21, 159-170. https: / / doi.org/10.1016/j. plrev.2017.07.002

Plassmann, H., O'Doherty, J., Shiv, B., \& Rangel, A. (2008). Marketing actions can modulate neural representations of experienced pleasantness. Proceedings of the National Academy of Sciences of the United States of America, 105(3), 1050-1054.https:/ /doi.org/10.1073/pnas.0706929105

Polanía, R., Woodford, M., \& Ruff, C. C. (2019). Efficient coding of subjective value. Nature Neuroscience, 22(1), 134-142. https: / / doi.org/10.1038/s41593-018-0292-0

Pool, E., Sennwald, V., Delplanque, S., Brosch, T., \& Sander, D. (2016). Measuring wanting and liking from animals to humans: A systematic review. Neuroscience and Biobehavioral Reviews, 63, 124-142. https://doi.org/10.1016/j.neubiorev.2016.01.006

Quoidbach, J., Gilbert, D. T., \& Wilson, T. D. (2013). The end of history illusion. Science, 339(6115), 96-98. https://doi.org/10.1126/science.1229294

Rampone, G., O' Sullivan, N., \& Bertamini, M. (2016). The role of visual eccentricity on preference for abstract symmetry. Plos One, 11(4), e0154428. https:/ / doi.org/10.1371/journal.pone. 0154428

Rao, R. P., \& Ballard, D. H. (1999). Predictive coding in the visual cortex: A functional interpretation of some extra-classical receptive-field effects. Nature Neuroscience, 2(1), 79-87. https://doi. org/10.1038/4580

Reber, R., Schwarz, N., \& Winkielman, P. (2004). Processing fluency and aesthetic pleasure: Is beauty in the perceiver's processing experience? Personality and Social Psychology Review, 8(4), 364-382. https://doi.org/10.1207/s15327957pspr0804\_3

Reber, R., Winkielman, P., \& Schwarz, N. (1998). Effects of perceptual fluency on affective judgments. Psychological Science, 9(1), 45-48. https://doi.org/10.1111/1467-9280.00008

Rhodes, G., Yoshikawa, S., Clark, A., Lee, K., McKay, R., \& Akamatsu, S. (2001). Attractiveness of facial averageness and symmetry in non-western cultures: In search of biologically based standards of beauty. Perception, 30(5), 611-625. https://doi.org/10.1068/p3123

Rhodes, G. (2006). The evolutionary psychology of facial beauty. Annual review of psychology, 57, 199-226. https://doi.org/10.1146/annurev.psych.57.102904.190208

Rutledge, R. B., Skandali, N., Dayan, P., \& Dolan, R. J. (2014). A computational and neural model of momentary subjective well-being. Proceedings of the National Academy of Sciences of the United States of America, 111(33), 12252-12257. https://doi.org/10.1073/pnas.1407535111

Ryali, C. K., Goffin, S., Winkielman, P., \& Yu, A. J. (2020). From likely to likable: The role of statistical typicality in human social assessment of faces. Proceedings of the National Academy of Sciences of the United States of America, 117(47), 29371-29380. https: / / doi.org /10.1073/ pnas.1912343117 
Salimpoor, V. N., Benovoy, M., Larcher, K., Dagher, A., \& Zatorre, R. J. (2011). Anatomically distinct dopamine release during anticipation and experience of peak emotion to music. Nature neuroscience, 14(2), 257-262.

Scarpi, D., Pizzi, G., \& Raggiotto, F. (2019). The extraordinary attraction of being ordinary: A moderated mediation model of purchase for prototypical products. Journal of Retailing and Consumer Services, 49, 267-278. https:/ /doi.org/10.1016/j.jretconser.2019.03.006

Schindler, I., Hosoya, G., Menninghaus, W., Beermann, U., Wagner, V., Eid, M., \& Scherer, K. R. (2017). Measuring aesthetic emotions: A review of the literature and a new assessment tool. Plos One, 12(6), e0178899. https://doi.org/10.1371/journal.pone.0178899

Schmidhuber, J. (2010). Formal theory of creativity, fun, and intrinsic motivation (1990-2010). IEEE transactions on autonomous mental development, 2(3), 230-247. https: / / doi.org / 10.1109/ $\{$ TAMD .2010 .2056368

Shany, O., Singer, N., Gold, B. P., Jacoby, N., Tarrasch, R., Hendler, T., \& Granot, R. (2019). Surpriserelated activation in the nucleus accumbens interacts with music-induced pleasantness. Social Cognitive and Affective Neuroscience, 14(4), 459-470. https://doi.org/10.1093/scan/ nsz019

Sherman, A., Grabowecky, M., \& Suzuki, S. (2015). In the working memory of the beholder: Art appreciation is enhanced when visual complexity is compatible with working memory. Journal of Experimental Psychology. Human Perception and Performance, 41(4), 898-903. https: //doi.org/10.1037/a0039314

Simonyan, K., \& Zisserman, A. (2015). Very deep convolutional networks for large-scale image recognition.

Skinner, B. F. (1975). The shaping of phylogenic behavior. Journal of the Experimental Analysis of Behavior, 24(1), 117-120.

Street, N., Forsythe, A. M., Reilly, R., Taylor, R., \& Helmy, M. S. (2016). A complex story: Universal preference vs. individual differences shaping aesthetic response to fractals patterns. Frontiers in Human Neuroscience, 10, 213. https://doi.org/10.3389/fnhum.2016.00213

Sutton, R. S. (1988). Learning to predict by the methods of temporal differences. Machine learning, 3(1), 9-44.

Sutton, R. S., \& Barto, A. G. (2018). Reinforcement learning: An introduction. MIT press.

Tinio, P. P. L., \& Leder, H. (2009). Just how stable are stable aesthetic features? symmetry, complexity, and the jaws of massive familiarization. Acta Psychologica, 130(3), 241-250. https: //doi.org/10.1016/j.actpsy.2009.01.001

Van de Cruys, S., Damiano, C., Boddez, Y., Król, M., Goetschalckx, L., \& Wagemans, J. (2021). Visual affects: Linking curiosity, aha-erlebnis, and memory through information gain. Cognition, 212, 104698. https://doi.org/10.1016/j.cognition.2021.104698

Van de Cruys, S., \& Wagemans, J. (2011). Putting reward in art: A tentative prediction error account of visual art. i-Perception, 2(9), 1035-1062. https://doi.org/10.1068/i0466aap

Van Geert, E., \& Wagemans, J. (2020). Order, complexity, and aesthetic appreciation. Psychology of aesthetics, creativity, and the arts, 14(2), 135-154. https://doi.org/10.1037/aca0000224

Vessel, E. A., Maurer, N., Denker, A. H., \& Starr, G. G. (2018). Stronger shared taste for natural aesthetic domains than for artifacts of human culture. Cognition, 179, 121-131. https://doi. org/10.1016/j.cognition.2018.06.009 
Vogel, T., Carr, E. W., Davis, T., \& Winkielman, P. (2018). Category structure determines the relative attractiveness of global versus local averages. Journal of Experimental Psychology. Learning, Memory, and Cognition, 44(2), 250-267. https://doi.org/10.1037/xlm0000446

Vogel, T., Ingendahl, M., \& Winkielman, P. (2020). The architecture of prototype preferences: Typicality, fluency, and valence. Journal of Experimental Psychology: General. https://doi.org/10. $1037 / x g e 0000798$

Wagner, V., Menninghaus, W., Hanich, J., \& Jacobsen, T. (2014). Art schema effects on affective experience: The case of disgusting images. Psychology of aesthetics, creativity, and the arts, 8(2), 120-129. https://doi.org/10.1037/a0036126

Weichselbaum, H., Leder, H., \& Ansorge, U. (2018). Implicit and explicit evaluation of visual symmetry as a function of art expertise. i-Perception, 9(2), 2041669518761464. https://doi.org/ $10.1177 / 2041669518761464$

Westphal-Fitch, G., Huber, L., Gómez, J. C., \& Fitch, W. T. (2012). Production and perception rules underlying visual patterns: Effects of symmetry and hierarchy. Philosophical Transactions of the Royal Society of London. Series B, Biological Sciences, 367(1598), 2007-2022. https: / / doi. org/10.1098/rstb.2012.0098

Winkielman, P., \& Cacioppo, J. T. (2001). Mind at ease puts a smile on the face: Psychophysiological evidence that processing facilitation elicits positive affect. Journal of Personality and Social Psychology, 81(6), 989-1000. https://doi.org/10.1037//0022-3514.81.6.989

Winkielman, P., Halberstadt, J., Fazendeiro, T., \& Catty, S. (2006). Prototypes are attractive because they are easy on the mind. Psychological Science, 17(9), 799-806. https://doi.org/10.1111/j. 1467-9280.2006.01785.x

Winkler, C., \& Rhodes, G. (2005). Perceptual adaptation affects attractiveness of female bodies. British Journal of Psychology, 96(Pt 2), 141-154. https://doi.org/10.1348/\{000712605X36343\}

Witek, M. A. G., Clarke, E. F., Wallentin, M., Kringelbach, M. L., \& Vuust, P. (2014). Syncopation, body-movement and pleasure in groove music. Plos One, 9(4), e94446. https://doi.org/10. 1371/journal.pone.0094446

Zaidel, D. W., \& Deblieck, C. (2007). Attractiveness of natural faces compared to computer constructed perfectly symmetrical faces. The International journal of neuroscience, 117(4), 423431. https://doi.org/10.1080/00207450600581928

Zajonc, R. B. (1968). Attitudinal effects of mere exposure. Journal of personality and social psychology, 9(2p2), 1.

Zajonc, R. B., Crandall, R., Kail Jr, R. V., \& Swap, W. (1974). Effect of extreme exposure frequencies on different affective ratings of stimuli. Perceptual and motor skills, 38(2), 667-678.

Zhang, J., Liu, X. L., So, M., \& Reder, L. M. (2020). Familiarity acts as a reduction in objective complexity. Memory \& Cognition, 48(8), 1376-1387. https:/ / doi.org/10.3758/s13421-02001055-z 


\section{Supplementary Material}

\section{Mere exposure effects}

In the main article, we report model fits assuming $n=2$ features. We also ran model fits for $n=1$. The resulting model fit as well as the underlying fit distributions and stimulus are shown in Figure 5. As opposed to the 2D-solution, the stimulus feature value in this case is such that it moves the mean of the system state away from the expected true distribution. The dynamics leading to the inverted-U-shape curve, however, remained the same for the $1 \mathrm{D}$ case. The initial increase in $A$ here is attributable to an increase in immediate sensory reward that outweighs the negative change in the system state's value. Eventually, the downturn in the development of $A$ over time is occasioned by the negative change in the system state's value outweighing the (high) immediate sensory reward. As in the 2D case, the relative weight of the change in the system state's value is higher than the one of immediate sensory reward in the 1D case.
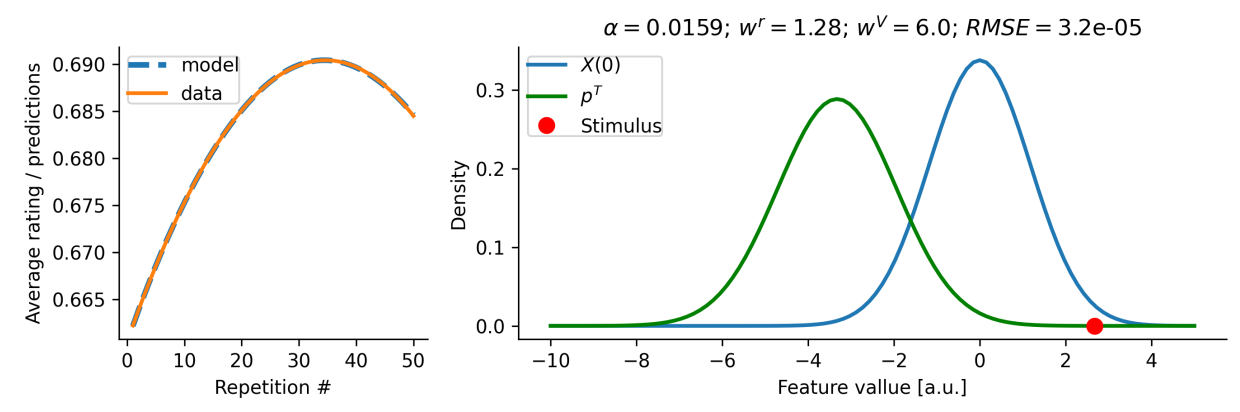

Figure 5: Best model fit for mere exposure effect with $n=1$ features. Left: Curve displaying the relationship between number of exposures and meta-analytic curve fits (solid orange lines) as well as model predictions (dashed blue lines). Right: Visualization of the system state $\mathbf{X}(0)$ (blue line), expected true distribution $p^{\mathrm{T}}$ (green line), and stimulus (red dot) for the best-fitting model.

The RMSE for the best-fitting 1D model was very slightly lower $\left(3.2 e^{-5}\right)$ than for the bestfitting 2D model. Relaxing the simplifying assumptions regarding the covariance matrix in the 2D case (equal variances, zero covariance) did not further lower the RMSE of the best-fitting 2D model. Given the very close match between data and model predictions, the small difference is probably not due to actual differences in model fit but precision in calculating model predictions. These results suggest that no complex, higher-dimensional stimulus representation is needed to model the average mere exposure effect across stimuli and observers.

\section{Symmetry, complexity, and familiarization}

In the main article, we report model fits given values for each stimulus category along two feature dimensions representing symmetry and complexity. We also explored whether the data reported by Tinio and Leder, 2009 can be fit assuming only one feature dimension. The best fit for this 1D case is shown in Figure 6. The RMSE for this variation was very close (only 0.001 higher) to the $2 \mathrm{D}$ case. It is noteworthy, however, that much like the model variations with set $w^{V}$ or set $w^{V}$ and $w^{r}$, the best 1D model's predictions tend to remain stable within one stimulus category more so than the empirical ratings. 

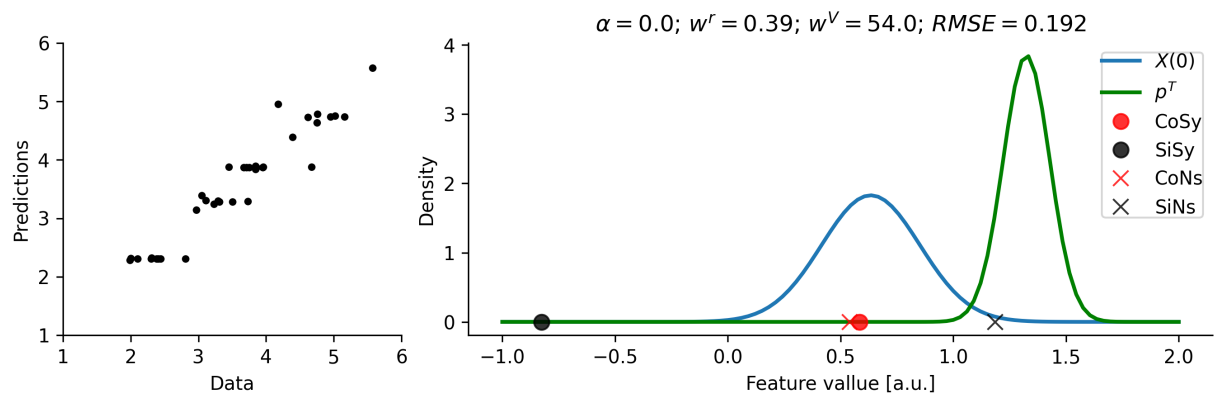

Figure 6: Best model fit for the data reported in Tinio and Leder, 2009 with $n=1$ features. Left: Model predictions vs. average ratings. Each dot represents one stimulus category in one familiarization condition. Right: Visualization of the system state $\mathbf{X}(0)$ (blue line) and expected true distribution $p^{T}$ (green line) for the best fitting model. Red symbols represent complex stimuli, black ones simple stimuli; circles represent symmetric stimuli, crosses non-symmetric stimuli.

In this one-dimensional case, complex (both symmetric and asymmetric) stimuli are represented as similar numbers that lie close to the mean of the initial system state's mean. Simplesymmetric stimuli are represented as a number far from the system state mean and even further from the expected true distribution's mean. Last, simple-non-symmetric stimuli are represented as lying between the means of the initial system state and the expected true distribution. The relative weight of immediate sensory reward is much lower than the weight of the change in the system state's value in the 1D case but it is not zero as in the 2D case presented in the main article. 